Document downloaded from:

http://hdl.handle.net/10251/45424

This paper must be cited as:

Merli Gisbert, R.; Lazaro, C.; Monleón Cremades, S.; Domingo Cabo, A. (2013). A molecular structural mechanics model applied to the static behavior of single-walled carbon nanotubes: New general formulation. Computers and Structures. 127:68-87. doi:10.1016/j.compstruc.2012.11.023.

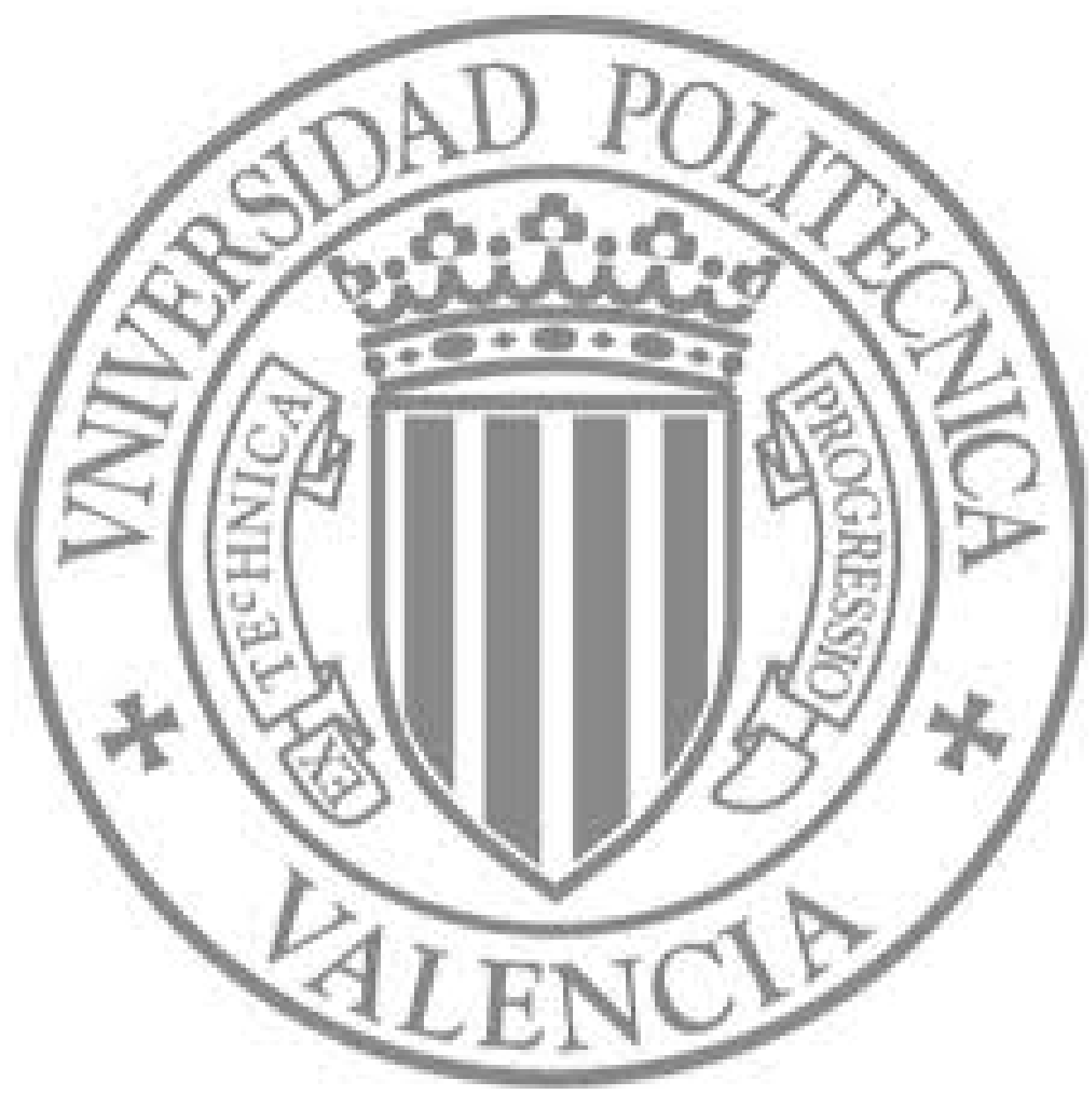

The final publication is available at

http://dx.doi:10.1016/j.compstruc.2012.11.023

Copyright Elsevier 


\title{
A Molecular Structural Mechanics model applied to the static behavior of single-walled Carbon nanotubes: New general formulation
}

\author{
R. Merli*a , C. Lázaro ${ }^{\mathrm{a}}$, S. Monleón ${ }^{\mathrm{a}}$, A. Domingo ${ }^{\mathrm{b}}$ \\ ${ }^{a}$ Departamento de Mecánica de los Medios Continuos y Teoría de Estructuras. \\ Escuela Técnica Superior de Ingenieros de Caminos, Canales y Puertos. \\ Universidad Politécnica de Valencia. \\ Camino de vera s/n, Valencia, Spain \\ ${ }^{b}$ Departamento de Ingeniería de la Construcción. \\ Escuela Técnica Superior de Ingenieros de Caminos, Canales y Puertos. \\ Universidad Politécnica de Valencia. \\ Camino de vera $s / n$, Valencia, Spain
}

\begin{abstract}
A new general formulation for the mechanical behavior of Single-Walled Carbon Nanotubes is presented. Carbon atoms are located at the nodes of an hexagonal honeycomb lattice wrapped into a cylinder. They are linked by covalent $C-C$ bonds represented by a truss or spring element, and the three-body interaction among two neighboring covalent bonds is reproduced by a rotational spring. The main advantage of our approach is to allow general load conditions (and any chirality) with no need of specific formulation for each load case, in contrast with previous works [26], [27], [31]. Four load configurations are adopted: tension, compression, bending and torsion of cantivelered SWCNTs. Calculations with our own codes for both AMBER and Morse potential functions have been carried out, aimed to compare their final results. Initial positions of the atoms (nodes) into nanotube cylindrical geometry has been reproduced in great detail by means of a conformal mapping from the planar graphene sheet. Therefore, the effect of initial SWCNTs curvature has been introduced explicitly through a system of initial stresses (prestressed state) which contribute to maintain their circular cross-section. Numerical results and deformed shapes for nanotubes with several diameters and chiralities under each load case are used to obtain their mechanical parameters with the only objective of checking the present formulation with previous works [28], [30], [20], [24]. Also, the significance of the atomistic discrete simulations at the nano-scale size against other continuum models is underlined.
\end{abstract}

Key words: Single-Walled Carbon Nanotubes, Molecular Structural Mechanics, AMBER potential, Morse potential, prestressed state, graphene sheet, conformal mapping

\section{Introduction}

Since their discovery [1], carbon nanotubes (CNTs) have attracted great interest of researchers and scientists because of their remarkable physical, mechanical and electrical properties [2]. For instance, CNTs show a singular coupling between mechanical strain and electrical conductivity [3, 4], becoming ideal candidates for making nanosensors and nano electro-mechanical systems (NEMS), with promising applications in robotics and biomechanics.

Regarding Materials Science and Engineering, nanotube reinforced composites and polymers have shown a wide range of potential applications, specially where a high ratio strength to weight is needed (e.g. aircraft industry). The main structural properties are their extreme longitudinal stiffness [5, 6, 7, 8] (Young's modulus $\simeq 1 \mathrm{TPa}$ ) and tensile strength [9, 10, 11] $\left(\sigma_{y} \simeq 50 \mathrm{GPa}\right)$.

Single-walled carbon nanotubes (SWCNTs) may be conceptualized as the result of rolling up a graphene sheet into a cylinder, and Multi-walled carbon nanotubes (MWCNTs) can be formed by placing several cylinders each one

Email addresses: ramergis@doctor.upv.es (R. Merli*), carlafer@mes.upv.es (C. Lázaro), smonleon@mes.upv.es (S. Monleón), adoming@mes.upv.es (A. Domingo) 
inside the rest coaxially, with a distance between walls of $0.34 \mathrm{~nm}$ as in bulk graphite. Each graphene sheet is formed by carbon atoms arranged in a covalent-bonded honeycomb lattice and the only link among carbon atoms of different sheets is the Van der Waals (VDW) interaction. Attending to the orientation of the hexagonal lattice with respect to the nanotube axis (chirality), three types of SWCNTs can be found:

1. ZigZag $(n, 0)$.- One third of the bonds are parallel to the tube axis.

2. Armchair $(n, n)$-- One third of the bonds are perpendicular to the tube axis.

3. Chiral $(n, m)$.- All of the bonds are oblique to the tube axis.

where $(n, m)$ are two integers which geometrically characterize the hexagonal lattice orientation into the cylinder (see [2]). The Zig-Zag and Armchair nanotubes are also called achiral and they are axisymmetric.

Covalent $C-C$ bonds can be modeled by using the Tersoff-Brenner potential [37, 38], which correctly corresponds with the Morse potential for strains below 10\% [9]. On the other hand, VDW interaction has been usually modeled by using the well-known 6-12 Lennard-Jones potential. More details for the potentials adopted in this work will be given in section 4

Previously to the practical applicability of nanotubes in manufacturing composites (as in other structural applications), a deep understanding of their mechanical behavior is needed. For this purpose, several experimental studies related to Multi-walled nanotubes [10, 12, 13, 14, 15] and to SWCNTs ropes [11, 16] have been carried out. However, to date, there is no experimental studies about individual SWCNTs due to their extremely small size and the subsequent difficulties in their manipulation. Therefore, theoretical work may be helpful for evaluating the structural response of SWCNTs.

Although many analytical or numerical methods applied to the mechanical behavior of nanotubes have been developed, they roughly fall into two different categories: atomistic scale and continuum scale methods. The atomistic methods (Molecular Dynamics, ab initio, tight-binding) can successfully reproduce physical phenomena as buckling [17, 18] and estimate elastic parameters of CNTs [3, 7], but they have the disadvantage of being limited at a relatively low number of atoms (about $10^{9}$ according to Wang et. al [19]) because of their high computational cost. Related to MD methods, the motion of a system of $\mathrm{N}$ particles is described dynamically by a vector of instantaneous positions and velocities (dimension $6 N$ ). In addition the time dependence implies using algorithms of time-integration sometimes complicated. Also, a difficult issue in MD is to describe the heat conduction with the surrounding media. Opposite to MD calculations, our MSM model have the following advantages:

1. No heat conduction is required to be accounted for in the formulation. Then, some reliability is sacrificed in order to simplify the model.

2. MSM models are basically static and no time-integration is needed.

3. For the same system size, only $3 N$ fundamental variables are involved (nodal displacements). This reduction implies a much higher numerical performance of the algorithm.

On the other hand, continuum methods are computationally cheaper and capable of analyse longer systems, but the choice of some parameters for establishing an equivalence with the atomistic level may be controversial. In fact, the wall thickness range from $t=0.066 \mathrm{~nm} \mathrm{[17]} \mathrm{to} \mathrm{the} \mathrm{usual} \mathrm{value} \mathrm{of} 0.34 \mathrm{~nm}$ [20] which corresponds to the interplanar distance in graphite. Even some authors deem CNTs as solid cylinders [19]. Moreover, continuum methods are insensitive to atomistic defects which have an important influence on the final response of CNTs.

Derived from Molecular Dynamic (MD) methods, a Molecular Structural Mechanics (MSM) model may be adopted, which is reasonable in terms of computational expense whereas atomistic scale is correctly displayed. Some previous works [21, 22, 24] considered CNTs as a frame system with carbon atoms located at nodes and rigid bars (provided with axial, flexural and torsional stiffnesses) representing covalent bonds. Different layers in MWCNTs were connected by several truss rods between neighboring atoms. Alternatively, Odegard et. al [23] modeled the graphene sheet as a 2D truss model with additional rods trough the unit hexagonal cell.

In the context of materials with granular structure at the microscopic level (microscale), lattice networks composed of Reissner's beam elements have been utilized in order to solve some conceptually related problems, as the dynamic fracture of a brittle material [25]. Hence, in the MSM framework, the use of Reissner beam elements with large rotations could be a promising extension for dealing with high local deformations into the nanotube wall.

Another kind of MSM model was called the 'stick-spiral' model by Chang and Gao [26] where covalent bonds were reproduced by axial springs and the three-body interaction was introduced directly by three spiral springs on 
each node. In that work (extended later in [27]), the influence of diameter and chirality in the Young's modulus and Poisson's ratio was analyzed for ZigZag and Armchair SWCNTs taking the curvature of the wall into account. The same subject was investigated by Natsuki et. al [28] but neglecting the curvature and similar issues were addressed by Wang [29]. Also Natsuki and Endo [30] extended their previous work to the tensile and compressive behavior of SWCNTs. All the aforementioned researches related to MSM have taken advantage of the axisymmetry of ZigZag and Armchair nanotubes, limiting their calculations to a small unit cell involving only a few atoms.

Moreover, Chang et. al [31] generalized the work in [26] to Chiral SWCNTs and extended it to the study of shear modulus. In a more general way, Meo and Rossi [20] implemented the 'stick-spiral' model in the commercial code ANSYS ${ }^{\circledR}$ and included Chiral SWCNTs in the study of the longitudinal behavior.

The main contributions of this paper can be outlined as follows:

1. In this paper, the main objective is to analyze the tensile, compressive, flexural and torsional behavior of SWCNTs by means of the 'stick-spiral' model and show the influence of the diameter and chirality in their mechanical response. For this purpose, analytical expressions have been derived through an energy approach and have been numerically implemented. The main advantage of our approach is the ability to reproduce general load conditions (and any chirality) with no need of additional equations, in contrast with previous works which treat specific load situations and chiralities with ad hoc equations for each case, and are limited to a small unit cell involving only a few atoms under a particular load case (see Chang and Gao [26], Xiao et. al [27], Natsuki et. al [28], Wang [29], Natsuki and Endo [30], Chang et. al [31]). This advantage translates into a higher versatility to deal with physically more realistic cases (e.g. the different chiralities and load configurations produced during the SWCNTs growing procedure).

2. A special issue not explicitly included in MSM models (although the wall-curvature was included in the equations) is the preenergy, defined as the excess of strain energy from an infinite planar graphene sheet to the nanotube [17, 32]. As has been shown [5, 33, 34, 35], this preenergy is proportional to the curvature of the wall $1 / R^{2}$ (where $\mathrm{R}$ is the tube radius) leading to an stabilization effect into its cross-sectional area. In this paper, we introduce the preenergy as a system of initial strains which produces a 'prestressed state' previous to the action of any external loading. Namely, the influence of this initial stresses is not negligible (differences around $5-15 \%$ in longitudinal stiffness are obtained), mainly in axial behavior of Single-Walled Carbon nanotubes (SWCNTs) as we can state from our results. Nonetheless, many released references simply ignore this fact.

3. A detailed comparison between the more usual interatomic potential functions (AMBER and Morse) has been performed under several load cases: tension, compression, bending and torsion. Likewise, both forcelengthening and moment-distortion nonlinear relationships have been regarded with Morse potential, generalizing the work of Meo and Rossi [20]. In this way, we keep the straightforward relationship between both interatomic potentials and both kinds of structural elements (bar elements and rotational springs) into the 'stickspiral' model. However, we have not included any geometrical updating into the iterative procedure.

4. Our numerical results have been compared with those from the standard beam models and we have concluded that the applicability of continuum methods is doubtful because they are unable to reproduce the atomistic detail at that nanoscale size. Moreover, the choice of some elastic (even geometrical) parameters is controversial and may vary from one load case to another. This conclusion is in disagreement with many research works (e.g. Wang et. al [19]), where continuum models are used.

The paper is organized as follows: in section 2 a schematic description of our model is provided. In section 3 the governing equations of our model are worked out. In section 4 a brief discussion about the adopted potentials is drawn. Details of the numerical implementation are given in section 5 Numerical results and some discussion of them are carried out in section 6 and finally, some concluding remarks are addressed in section 7 .

\section{Conceptual description of the model}

A schematic description of the present MSM model is depicted in figure1 for a ZigZag SWCNT. Covalent bonds are represented by 'bar' (or 'truss') elements which can just deform under axial strain and the three-body interaction is represented by rotational springs which only resist against angular distortion among two neighboring covalent bonds 
on each node. Exclusively linear displacements are taken into account at each node, therefore angular distortions will be written depending on them. All loads are applied at the nodes.

Note that such a 3D model is able to reproduce general loading states (tensile, compressive, flexural loading) and there is no need to derive particular expressions for each case as in most of previous researches (e.g. [26, 27]).

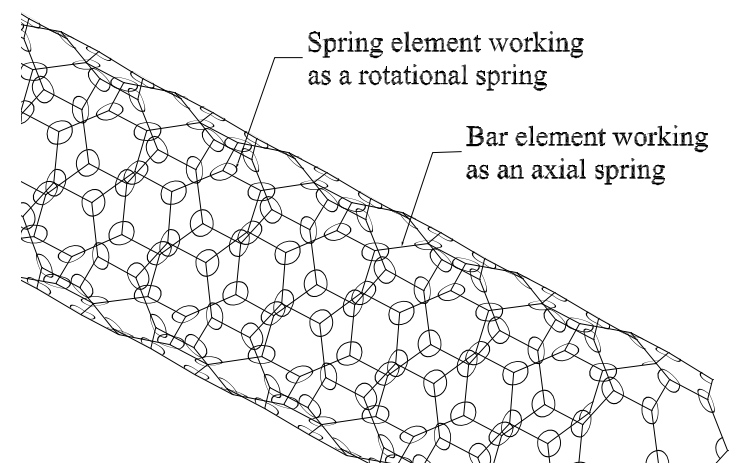

Figure 1: Geometrical description of elements

\section{Governing equations}

\subsection{Kinematic equations}

\subsubsection{Bar element}

We can define the axial strain among two bonded atoms as the change in length of the covalent bond and it can be written as:

$$
\epsilon^{a}=\Delta l=\left(\lambda^{a}\right)^{T} \cdot\left(\mathbf{u}_{j}-\mathbf{u}_{i}\right)=\left[-\left(\lambda^{a}\right)^{T} \quad\left(\lambda^{a}\right)^{T}\right]\left[\begin{array}{l}
\mathbf{u}_{j} \\
\mathbf{u}_{i}
\end{array}\right]=\left[\begin{array}{ll}
\mathbf{C}_{i}^{a} & \mathbf{C}_{j}^{a}
\end{array}\right]\left[\begin{array}{l}
\mathbf{u}_{j} \\
\mathbf{u}_{i}
\end{array}\right]=\mathbf{C}_{l}^{a} \mathbf{u}^{a}
$$

where:

$$
\begin{array}{ll}
\epsilon^{a}=\Delta l= & \text { axial strain along the bar element. } \\
\lambda^{a}= & \text { unit vector along the element. } \\
\mathbf{u}_{i}, \mathbf{u}_{j}= & \text { nodal displacements. } \\
\mathbf{x}_{i}, \mathbf{x}_{j}= & \text { nodal vectors undeformed shape. } \\
\mathbf{x}_{i}^{\prime}, \mathbf{x}_{j}^{\prime}= & \text { nodal vectors deformed shape. } \\
\mathbf{C}_{l}^{a}= & \text { linear kinematic matrix of the bar element }
\end{array}
$$

Note that eq.(1) just gives the axial strain in terms of the difference between the nodal displacements (three DOFs each) projected onto the initial direction of the element.

\subsubsection{Rotational spring element}

Three-body interactions are represented in the present model by in-plane rotational springs involving three neighboring carbon atoms as can be seen in figure 3 where bar elements are not included but their directions are depicted by auxiliary straight lines.

The angular strain in the spring element is defined as the change in angle involved among three neighboring atoms (or nodes):

$$
\epsilon^{1}=\Delta \gamma^{a b}=A-\alpha
$$

Taking cosines in (2) and assuming the displacements are small, we can write:

$$
\epsilon^{1}=\frac{\cos \alpha-\cos A}{\sin A}=\frac{\left[\left(\lambda^{a^{\prime}}\right)^{T} \lambda^{b^{\prime}}\right]-\left[\left(\lambda^{a}\right)^{T} \lambda^{b}\right]}{\sin A}
$$




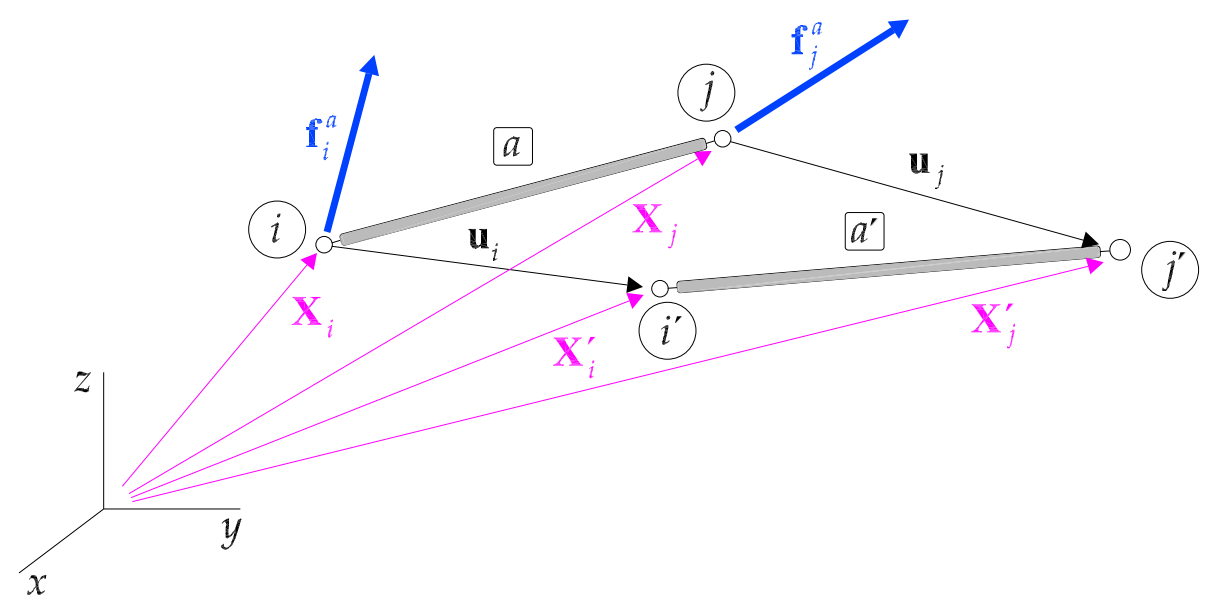

Figure 2: Forces and displacements at the bar ends

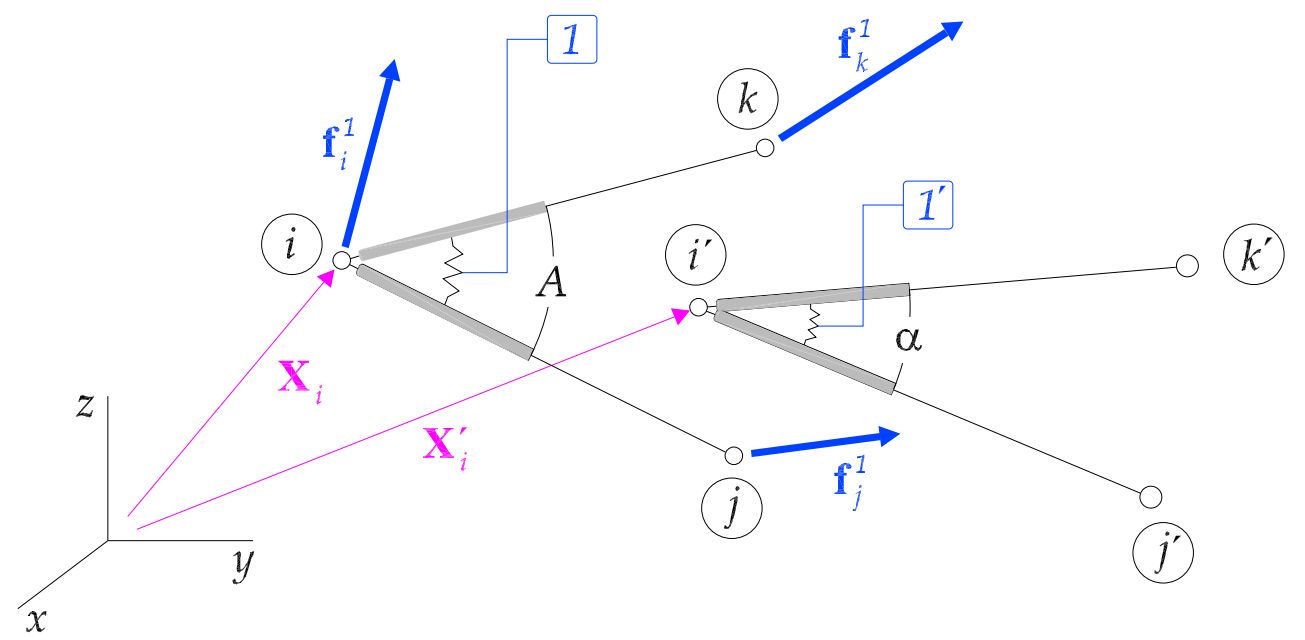

Figure 3: Forces and displacements acting on the spring element

where ()$^{\prime}$ denotes the variable is associated to the deformed shape. The unit vectors $\lambda^{a^{\prime}}, \lambda^{b^{\prime}}$ may be written in terms of

$$
\begin{aligned}
& \lambda^{a^{\prime}}=\frac{1}{l^{a^{\prime}}}\left\{l^{a} \lambda^{a}+\mathbf{u}_{j}-\mathbf{u}_{i}\right\}=\frac{1}{l^{a^{\prime}}}\left\{l^{a} \lambda^{a}+\mathbf{u}_{i j}\right\} \\
& \lambda^{b^{\prime}}=\frac{1}{l^{b^{\prime}}}\left\{l^{b} \lambda^{b}+\mathbf{u}_{k}-\mathbf{u}_{i}\right\}=\frac{1}{l^{b^{\prime}}}\left\{l^{b} \lambda^{b}+\mathbf{u}_{i k}\right\}
\end{aligned}
$$

where:

$A=\quad$ initial angle between three neighboring atoms.

$\alpha=\quad$ final angle between three neighboring atoms.

$l^{a}, l^{b}=\quad$ initial lengths of bar elements.

$l^{a^{\prime}}, l^{b^{\prime}}=$ final lengths of bar elements.

Regarding (4) and neglecting terms of second order, $\cos \alpha$ is given by:

$$
\cos \alpha=\frac{l^{a} l^{b}\left(\lambda^{a}\right)^{T} \lambda^{b}+l^{a}\left(\lambda^{a}\right)^{T} \mathbf{u}_{i k}+l^{b}\left(\lambda^{b}\right)^{T} \mathbf{u}_{i j}}{l^{a} l^{b}+b^{a} \Delta l^{b}+l^{b} \Delta l^{a}}
$$


Substituting (5) (only keeping the first term in the denominator) in (3) and rearranging terms, the following expression can be obtained:

$$
\epsilon^{1}=\left[\begin{array}{lll}
\left(\boldsymbol{\Gamma}_{j}^{1}\right)^{T} & \left(\boldsymbol{\Gamma}_{i}^{1}\right)^{T} & \left(\boldsymbol{\Gamma}_{k}^{1}\right)^{T}
\end{array}\right]\left[\begin{array}{l}
\mathbf{u}_{j} \\
\mathbf{u}_{i} \\
\mathbf{u}_{k}
\end{array}\right]=\left[\begin{array}{lll}
\mathbf{C}_{j}^{1} & \mathbf{C}_{i}^{1} & \mathbf{C}_{k}^{1}
\end{array}\right]\left[\begin{array}{c}
\mathbf{u}_{j} \\
\mathbf{u}_{i} \\
\mathbf{u}_{k}
\end{array}\right]=\mathbf{C}_{l}^{1} \mathbf{u}^{1}
$$

where:

$$
\begin{aligned}
& \boldsymbol{\Gamma}_{j}^{1}=\left[\frac{\lambda^{b}}{l^{a} \sin A}-\frac{\lambda^{a}}{l^{a} \tan A}\right] \\
& \boldsymbol{\Gamma}_{k}^{1}=\left[\frac{\lambda^{a}}{l^{b} \sin A}-\frac{\lambda^{b}}{l^{b} \tan A}\right] \\
& \boldsymbol{\Gamma}_{i}^{1}=-\boldsymbol{\Gamma}_{j}^{1}-\boldsymbol{\Gamma}_{k}^{1}
\end{aligned}
$$

and $\mathbf{C}_{l}^{1}$ means the linear kinematic matrix of the spring element.

\subsubsection{Whole structural system}

This section refers to any group of bar and spring elements properly assembled. In particular, to those systems which reproduce SWNTs geometry as can be seen in figure 1 For the sake of clarity, the next parameters are defined:

$\mathbf{u}_{R}=$ displacements of free nodes.

$\mathbf{u}_{A}=$ displacements of fixed (constrained) nodes. Herein $\mathbf{u}_{A}=\mathbf{0}$ but displacements could be imposed with non-zero values.

$\boldsymbol{\epsilon}^{\lambda}=$ vector of axial strains.

$\boldsymbol{\epsilon}^{\Gamma}=$ vector of angular distortions.

$\mathbf{C}^{\lambda}=$ kinematic matrix associated to axial strains

$\mathbf{C}^{\Gamma}=$ kinematic matrix associated to angular strains

Equations (1) and (6) can be grouped and rearranged for the whole system in the following way:

$$
\left[\begin{array}{l}
\boldsymbol{\epsilon}^{\lambda} \\
\boldsymbol{\epsilon}^{\Gamma}
\end{array}\right]=\left[\begin{array}{l}
\mathbf{C}^{\lambda} \\
\mathbf{C}^{\Gamma}
\end{array}\right][\mathbf{u}]=\left[\begin{array}{ll}
\mathbf{C}_{R}^{\lambda} & \mathbf{C}_{A}^{\lambda} \\
\mathbf{C}_{R}^{\Gamma} & \mathbf{C}_{A}^{\Gamma}
\end{array}\right]\left[\begin{array}{l}
\mathbf{u}_{R} \\
\mathbf{u}_{A}
\end{array}\right]
$$

where the kinematic matrices defined above are formed by the corresponding blocks of $\mathbf{C}_{l}^{i}, \quad i=a, b, c, \ldots$ or $\mathbf{C}_{l}^{j}$, $j=1,2,3, \ldots$ depending on the element considered. Regarding $\mathbf{u}_{A}$ vanishes in the present work, eq. (8) is reduced to:

$$
\begin{aligned}
\boldsymbol{\epsilon}^{\lambda} & =\mathbf{C}_{R}^{\lambda} \mathbf{u}_{R} \\
\boldsymbol{\epsilon}^{\Gamma} & =\mathbf{C}_{R}^{\Gamma} \mathbf{u}_{R}
\end{aligned}
$$

\subsection{Equilibrium equations}

\subsubsection{Bar element}

The Virtual Work Equation (VWE) associated to any bar element (see fig 2) can be expressed:

$$
\left(\mathbf{f}_{i}^{a}\right)^{T} \delta \mathbf{u}_{i}+\left(\mathbf{f}_{j}^{a}\right)^{T} \delta \mathbf{u}_{j}=n^{a} \delta \epsilon^{a}
$$

For all kinematically admissible virtual displacements $\delta \mathbf{u}_{i}, \delta \mathbf{u}_{j}$. Where:

$\mathbf{f}_{i}^{a}, \mathbf{f}_{j}^{a}=$ fraction of the external forces contributing to axial strain

$n^{a}=\quad$ axial force

$\delta \epsilon^{a}=\quad$ virtual axial strain.

Substituting (1) in (10) yields:

$$
\begin{aligned}
{\left[\begin{array}{ll}
\mathbf{f}_{i}^{a} & \mathbf{f}_{j}^{a}
\end{array}\right]\left[\begin{array}{l}
\delta \mathbf{u}_{i} \\
\delta \mathbf{u}_{j}
\end{array}\right] } & =n^{a}\left[\begin{array}{ll}
\mathbf{C}_{i}^{a} & \mathbf{C}_{j}^{a}
\end{array}\right]\left[\begin{array}{l}
\delta \mathbf{u}_{i} \\
\delta \mathbf{u}_{j}
\end{array}\right] \\
\left(\mathbf{f}^{\mathbf{a}}\right)^{T} \delta \mathbf{u}^{a} & =n^{a} \mathbf{C}_{l}^{a} \delta \mathbf{u}^{a}
\end{aligned}
$$


therefore:

$$
\mathbf{f}^{\mathbf{a}}=n^{a}\left(\mathbf{C}_{l}^{a}\right)^{T}=n^{a} \mathbf{H}_{l}^{a}
$$

where $\mathbf{H}_{l}^{a}$ denotes the linear equilibrium matrix of the bar element. From (12) it is clear that the contragradience relationship works strictly.

\subsubsection{Rotational spring element}

Similarly to (10), the VWE associated to any spring element (see fig 3) can be written:

$$
\left(\mathbf{f}_{j}^{1}\right)^{T} \delta \mathbf{u}_{j}+\left(\mathbf{f}_{i}^{1}\right)^{T} \delta \mathbf{u}_{i}+\left(\mathbf{f}_{k}^{1}\right)^{T} \delta \mathbf{u}_{k}=m^{1} \delta \epsilon^{1}
$$

For all group of virtual displacements $\delta \mathbf{u}_{j}, \delta \mathbf{u}_{i}, \delta \mathbf{u}_{k}$ kinematically admissible. Where:

$\mathbf{f}_{j}^{1}, \mathbf{f}_{i}^{1}, \mathbf{f}_{k}^{1}=$ fraction of the external forces contributing to angular strain

$m^{1}=\quad$ moment force

$\delta \epsilon^{1}=\quad$ virtual angular strain.

Substituting (6) in (13) the contragradience relationship equivalent to (12) is obtained:

$$
\mathbf{f}^{1}=m^{1}\left(\mathbf{C}_{l}^{1}\right)^{T}=m^{1} \mathbf{H}_{l}^{1}
$$

where $\mathbf{H}_{l}^{1}$ means the linear equilibrium matrix of the spring element.

\subsubsection{Whole structural system}

The energy equilibrium approach in terms of the external forces at free nodes $\overline{\mathbf{f}}_{R}$ can be written:

$$
\overline{\mathbf{f}}_{R}^{T} \delta \mathbf{u}_{R}=\mathbf{n}^{T} \delta \boldsymbol{\epsilon}^{\lambda}+\mathbf{m}^{T} \delta \boldsymbol{\epsilon}^{\Gamma}
$$

Taking first variations in (9) and substituting in (15):

$$
\overline{\mathbf{f}}_{R}^{T} \delta \mathbf{u}_{R}=\mathbf{n}^{T} \mathbf{C}_{R}^{\lambda} \delta \mathbf{u}_{R}+\mathbf{m}^{T} \mathbf{C}_{R}^{\Gamma} \delta \mathbf{u}_{R}
$$

Equating coefficients in (16) and transposing, yields:

$$
\overline{\mathbf{f}}_{R}=\left[\begin{array}{ll}
\left(\mathbf{C}_{R}^{\lambda}\right)^{T} & \left(\mathbf{C}_{R}^{\Gamma}\right)^{T}
\end{array}\right]\left[\begin{array}{l}
\mathbf{n} \\
\mathbf{m}
\end{array}\right]=\left[\begin{array}{ll}
\mathbf{H}_{R}^{\lambda} & \mathbf{H}_{R}^{\Gamma}
\end{array}\right]\left[\begin{array}{l}
\mathbf{n} \\
\mathbf{m}
\end{array}\right]=\mathbf{H}_{R} \mathbf{p}
$$

where:

$\mathbf{n}=\quad$ vector of internal axial forces

$\mathbf{m}=\quad$ vector of internal moment forces

$\mathbf{p}=\quad$ vector of internal forces

$\mathbf{H}_{R}=$ reduced equilibrium matrix of the whole system (verifying contragradience).

It is worth noting that eq.(17) can also be expressed as:

$$
\overline{\mathbf{f}}_{R}=\mathbf{H}_{R}^{\lambda} \mathbf{n}+\mathbf{H}_{R}^{\Gamma} \mathbf{m}=\overline{\mathbf{f}}_{R}^{\lambda}+\overline{\mathbf{f}}_{R}^{\Gamma}
$$

which shows that $\overline{\mathbf{f}}_{R}$ can be obtained as the sum of the fraction corresponding to axial forces and the fraction corresponding to moment forces.

\subsection{Constitutive equations}

The strain-internal force relationship for any group of properly assembled elements takes the form:

$$
\boldsymbol{\epsilon}=\boldsymbol{\epsilon}_{0}+\mathbf{F p}
$$


where $\epsilon_{0}$ is referred to as the initial strains (preenergy) related to the change in shape from the graphene sheet to the SWCNT, and $\mathbf{F}$ is a diagonal matrix involving the flexibility parameters of each element. The reverse expression is given by:

$$
\mathbf{p}=\mathbf{F}^{-1} \boldsymbol{\epsilon}-\mathbf{F}^{-1} \epsilon_{0}=\mathbf{K} \boldsymbol{\epsilon}-\mathbf{K} \epsilon_{0}=\mathbf{K} \boldsymbol{\epsilon}+\mathbf{p}_{0}
$$

Obviously, $\mathbf{K}$ is a diagonal matrix involving the stiffness parameters derived from the interatomic potential (see section (4) and $\mathbf{p}_{0}$ are the initial forces consistent with $\boldsymbol{\epsilon}_{0}$. Splitting in blocks, eq. (20) becomes:

$$
\left[\begin{array}{l}
\mathbf{n} \\
\mathbf{m}
\end{array}\right]=\left[\begin{array}{cc}
\mathbf{K}^{\lambda} & \mathbf{0} \\
\mathbf{0} & \mathbf{K}^{\Gamma}
\end{array}\right]\left[\begin{array}{l}
\boldsymbol{\epsilon}^{\lambda} \\
\boldsymbol{\epsilon}^{\Gamma}
\end{array}\right]+\left[\begin{array}{l}
\mathbf{n}_{0} \\
\mathbf{m}_{0}
\end{array}\right]
$$

Since the nanotube geometry has been generated through a conformal mapping from a graphene sheet to a cylinder where carbon atoms are kept into the surface and covalent bonds are located along the respective secant among two carbon atoms, initial longitudinal strains have been calculated as the difference in length from the graphene sheet to the cylinder. Also, initial angular distortions has been obtained as the difference in angle from graphene to the nanotube.

\subsection{Stiffness equations}

In order to obtain the stiffness equation of the whole structural system, we substitute eqs.(21) and (9) in eq.(17), so:

$$
\overline{\mathbf{f}}_{R}=\left[\begin{array}{ll}
\mathbf{H}_{R}^{\lambda} & \mathbf{H}_{R}^{\Gamma}
\end{array}\right]\left[\begin{array}{cc}
\mathbf{K}^{\lambda} & \mathbf{0} \\
\mathbf{0} & \mathbf{K}^{\Gamma}
\end{array}\right]\left[\begin{array}{l}
\left(\mathbf{H}_{R}^{\lambda}\right)^{T} \\
\left(\mathbf{H}_{R}^{\Gamma}\right)^{T}
\end{array}\right] \mathbf{u}_{R}+\left[\begin{array}{ll}
\mathbf{H}_{R}^{\lambda} & \mathbf{H}_{R}^{\Gamma}
\end{array}\right]\left[\begin{array}{l}
\mathbf{n}_{0} \\
\mathbf{m}_{0}
\end{array}\right]
$$

operating:

$$
\begin{gathered}
\overline{\mathbf{f}}_{R}=\left[\mathbf{H}_{R}^{\lambda} \mathbf{K}^{\lambda}\left(\mathbf{H}_{R}^{\lambda}\right)^{T}+\mathbf{H}_{R}^{\Gamma} \mathbf{K}^{\Gamma}\left(\mathbf{H}_{R}^{\Gamma}\right)^{T}\right] \mathbf{u}_{R}+\mathbf{H}_{R} \mathbf{p}_{0} \\
\overline{\mathbf{f}}_{R}=\widetilde{\mathbf{K}}_{R} \mathbf{u}_{R}+\mathbf{f}_{0}
\end{gathered}
$$

where:

$\widetilde{\mathbf{K}}_{R}=$ reduced stiffness matrix

$\mathbf{f}_{0}=\quad$ effect of initial forces at free nodes (prestressed state).

Although it has not been shown in this paper, a similar procedure could be performed to achieve individual stiffness matrices of bar and spring elements. Since the equilibrium matrix $\mathbf{H}_{R}$ is formed from the corresponding blocks of individual equilibrium matrices of each element (assembly of elements), it is easy to prove that $\widetilde{\mathbf{K}}_{R}$ can be obtained assembling individual stiffness matrices in the usual sense, which constitutes a great advantage for its numerical implementation compared with other published works [26, 31].

\section{Interatomic potentials}

The axial and angular stiffness parameters (so-called constant forces) represent the interaction between carbon atoms into the nanotube, which is usually introduced in theoretical MSM models by means of a potential function $U$. This function and the stiffness parameters are related by:

$$
K^{\lambda}=\frac{\partial^{2} U}{\partial(\Delta r)^{2}} \quad K^{\Gamma}=\frac{\partial^{2} U}{\partial(\Delta \theta)^{2}}
$$

where:

$\Delta r=$ difference in length of covalent bonds from their equilibrium position

$\Delta \theta=$ difference in angle among neighboring covalent bonds from their equilibrium angle

$K^{\lambda}=$ axial stiffness of any bar element

$K^{\Gamma}=$ angular stiffness of any spring element

Potential functions usually employed in molecular mechanics can be classified into two main categories: 
1. Harmonic potentials.- The potential is described as the addition of the potentials due to different relative displacements between atoms, expressed as a quadratic form each one:

$$
U=\sum U_{r}+\sum U_{\theta}+\sum U_{\phi}+\sum U_{\omega}+\sum U_{v d w}
$$

where:

$U_{r}=\quad$ potential including the change in length of the bond

$U_{\theta}=$ potential including the change in angle among neighboring covalent bonds

$U_{\phi}=\quad$ potential due to the torsion of the bond

$U_{\omega}=$ potential due to the torsion of the bond (out-of-plane rotation)

$U_{v d w}=$ potential including Van der Waals (VDW) interaction

Usually, definition [25] is simplified [26, 28, 29, 36] by only keeping the first and second terms as:

$$
U=\sum \frac{1}{2} k_{r}(\Delta r)^{2}+\sum \frac{1}{2} k_{\theta}(\Delta \theta)^{2}
$$

where $k_{r}, k_{\theta}$ are the force constants to the longitudinal and angular variations. The first sum is extended over all covalent bonds and the second over all angles among bonds.

On the other hand, if the MSM model is constituted with frame elements (e.g. [21, 22, 24]) also the third and fourth terms are retained and grouped into a quadratic form associated to the torsion of the bar element.

In this paper, eq. 26) with AMBER 1 constants are adopted. Regarding eqs.(24) and following [21], [24] and [28], the next values are adopted:

$$
K^{\lambda}=k_{r}=652 \frac{n N}{\text { bond } \cdot n m} \quad K^{\Gamma}=k_{\theta}=0.876 \frac{n N \cdot n m}{\text { bond } \cdot \text { rad }}
$$

2. Multi-body potentials.- The so-called REBO2 potentials involve the effect of the rest of atoms in the covalent bond interaction. Therefore, the two-body terms in the potential function will include the distance among the two atoms regarded and the angles formed with neighboring covalent bonds. The most extensively used (e.g. [17, 18, 32, 35, 39, 40]) potential function (mainly in Molecular Dynamics simulations) is the Tersoff-Brenner (TB) potential [37, 38]. However, its formulation is relatively complicated for further numerical implementation due to effects of variation in length and angle are coupled, hence can be approached by the Morse potential [9, 30] function for longitudinal strains below $10 \%$, which is given by:

$$
\begin{aligned}
E & =\sum E_{r}+\sum E_{\theta} \\
E_{r} & =D_{e}\left\{\left[1-e^{-\beta(\Delta r)}\right]^{2}-1\right\} \\
E_{\theta} & =\frac{1}{2} k_{\theta}(\Delta \theta)^{2}\left[1+k_{s}(\Delta \theta)^{4}\right]
\end{aligned}
$$

where the parameters involved take the following values [9]:

$$
\begin{array}{rlrl}
D_{e} & =0.2895 \mathrm{nN} \cdot \mathrm{nm} & & \beta=38.43 \mathrm{~nm}^{-1} \\
k_{\theta} & =0.8998 \mathrm{nN} \cdot \mathrm{nm} & k_{s}=0.754 \mathrm{rad}^{-4}
\end{array}
$$

Moreover, $\Delta r$ is the change in length of covalent bonds from their initial distance of equilibrium in the nanotube that is around $0.142 \mathrm{~nm}$, and $\Delta \theta$ is the change in angle from the initial one in the SWCNT which is about $2 \pi / 3$. Both values depend on the chirality and vary from one bond to another.

As can be seen from eqs. (28) to (30) the contribution of variations in length and angle are uncoupled. In this case, definitions (24) take the form:

$$
\begin{aligned}
& K^{\lambda}=2 \beta^{2} D_{e} e^{-\beta(\Delta r)}\left[2 e^{-\beta(\Delta r)}-1\right] \\
& K^{\Gamma}=k_{\theta}\left[1+15 k_{s}(\Delta \theta)^{4}\right]
\end{aligned}
$$

which definitely leads to a numerical iterative procedure (section 5).

\footnotetext{
${ }^{1}$ Assisted Model Building with Energy Refinement, force field well-known in bio-molecular simulation

${ }^{2}$ Reactive Empirical Bond Order
} 
In order to qualitatively compare both potentials we depict the force-lengthening relation in fig $4(\mathrm{a})$ and the moment-distortion relation in fig 4(b), given by:

$$
\begin{aligned}
& n=2 \beta D_{e} e^{-\beta(\Delta r)}\left[1-e^{-\beta(\Delta r)}\right] \\
& m=k_{\theta}(\Delta \theta)\left[1+3 k_{s}(\Delta \theta)^{4}\right]
\end{aligned}
$$

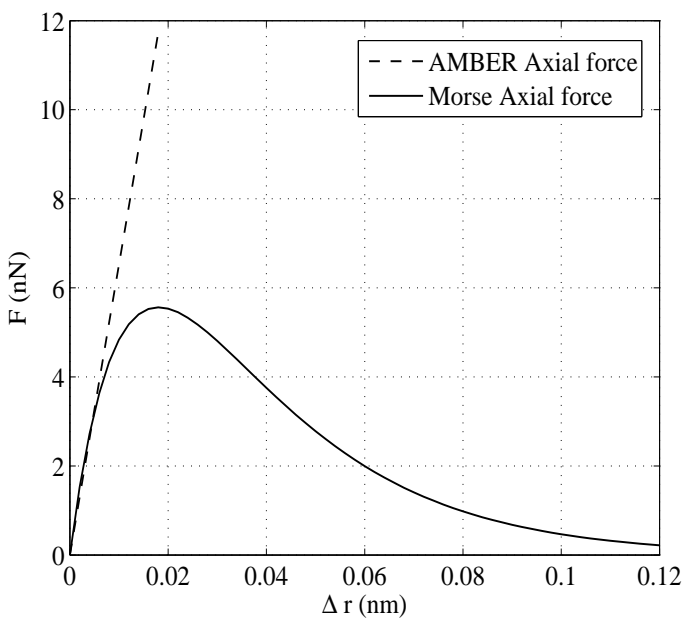

(a) Force-length variation

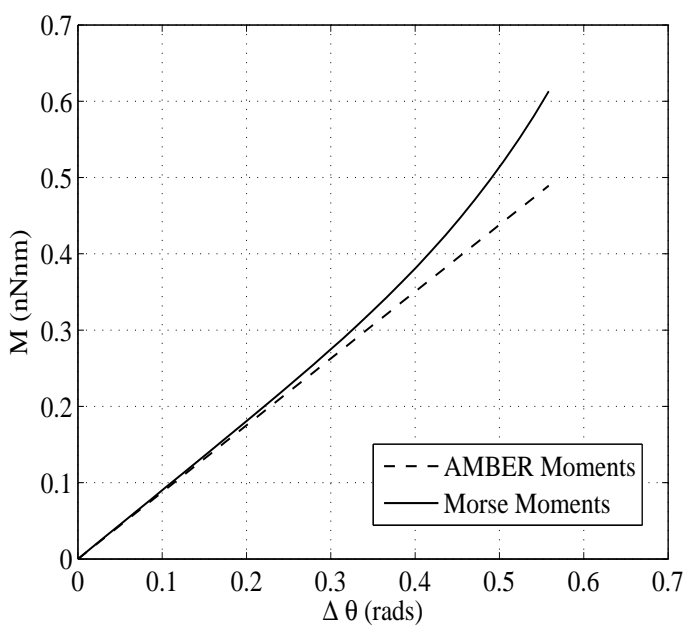

(b) Moment-angle variation

Figure 4: Comparison AMBER and Morse potentials

From their values, can be intuitively concluded that axial stiffness has higher influence than angular stiffness on the mechanical response of SWCNTs. Hence Meo and Rossi [20] used the Morse potential for axial stiffness but linearized angular stiffness. Anyway, from fig 4(a) is expected that the choice of the potential will be inconsequential as far as axial strains remain lower than $0.01 \mathrm{~nm}$ (about $7 \%$ ).

\section{Numerical implementation}

General SWCNTs geometry has been generated by means of a conformal mapping from the graphene sheet to the cylinder surface. Carbon atoms are kept on the surface and covalent bonds are located along secants among two covalent-bonded atoms. This geometry as well as the output drawing results has been programmed in VisualLISP code. On the other hand, implementation of the governing equations has been done in $\mathrm{C}++$ programming language.

Two main subjects are treated: First, importance of the choice of the potential function on the final response of nanotubes is investigated. Secondly, influence of the insertion of initial forces (prestressed state) with each potential is deemed. Thus, four different situations has been coded.

\subsection{AMBER potential}

Regarding constant stiffness for each element (eq. (27)) is the most easy way to introduce the interatomic interaction into the code and leads to the simple (non-iterative) flow diagram depicted in fig. 5

Two codes have been developed with AMBER potential (one with and one without initial forces) for seeking the influence of the prestressed state in SWCNTs response. This initial forces has been introduced through second terms in eqs. 21) and (23). Nevertheless, diagram in fig. 5is valid for both calculations. 


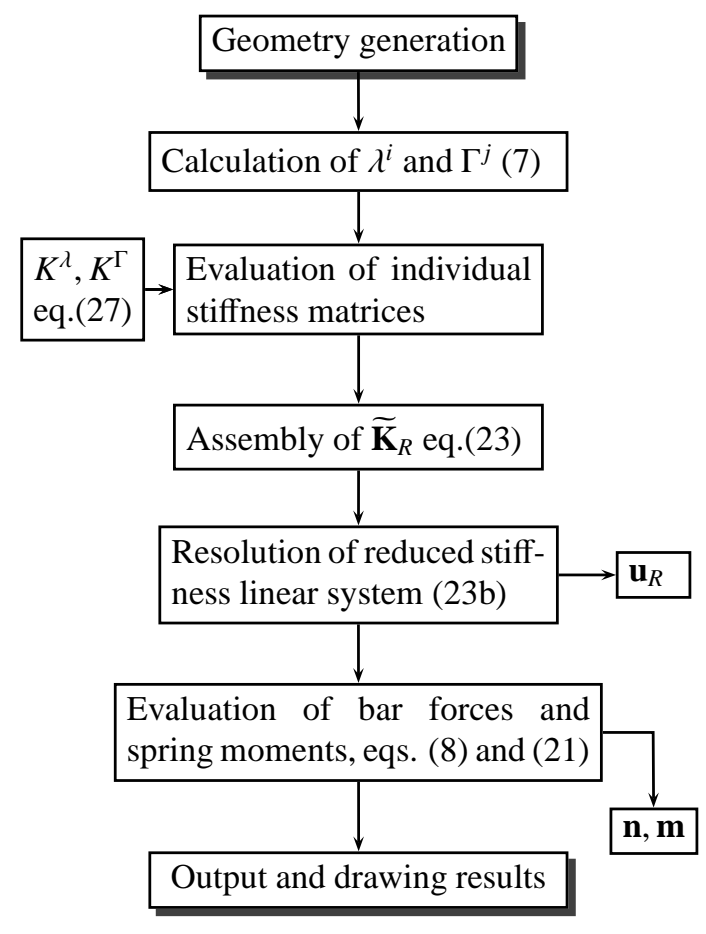

Figure 5: Simple flow diagram with AMBER potential

\subsection{Morse potential}

Introduction of the Morse potential in the governing equations is essentially different from the previous case. In fact, equations (32) and (33) are tangent stiffnesses to the equilibrium path of each element and they depend on the SWCNT deformed shape at each point of these trajectories. This variable nature of stiffness can be called constitutive nonlinearity and leads to the iterative procedure outlined in figure 6, based in the so-called Newton-like methods for solving nonlinear problems numerically. Superscripts $k$ indicate the iteration counter and subscripts $i, j$ the bar and spring element respectively.

Denoting $u_{\rho}$ as a generic component of $\mathbf{u}_{R}$ at the beginning of each increment, let us define the absolute error in the $\rho$-component of $\mathbf{u}_{R}$ as:

$$
E_{\rho}=\left|\Delta u_{\rho}\right| \quad \rho=1,2, \ldots, n
$$

where $n$ is the total number of DOFs involved in the reduced stiffness linear system. Then, convergence criteria adopted has been:

$$
\left\|\Delta \mathbf{u}_{R}\right\|=\max \left\{E_{\rho}\right\}<\varepsilon=10^{-6}
$$

Note that $K^{\lambda^{0}}, K^{\Gamma^{0}}$ are the tangent stiffness parameters for the undeformed shape of the nanotube. If prestresses are not taken into account $\Delta r=\Delta \theta=0$ will be substituted in eqs. (32) and (33) but if prestresses are included $\Delta r=\Delta r_{0}$ and $\Delta \theta=\Delta \theta_{0}$ (initial strains from the graphene sheet) will be employed instead.

Also, it should be pointed out that $\left(\mathbf{K}^{\lambda}\right)_{i}^{k},\left(\mathbf{K}^{\Gamma}\right)_{j}^{k}$ are exclusively updated through $\left(K^{\lambda}\right)_{i}^{k},\left(K^{\Gamma}\right)_{j}^{k}($ constitutive updating), since the vectors $\lambda^{i}, \Gamma^{j}$ involved are taken from the undeformed shape (geometrical linear analysis), correspondingly with the small displacements assumption. Further improvements of our formulation as geometrical nonlinearity (where $\lambda^{i}, \Gamma^{j}$ are suitably updated iteratively) are currently in progress and values of critical stresses and strains will be given. 


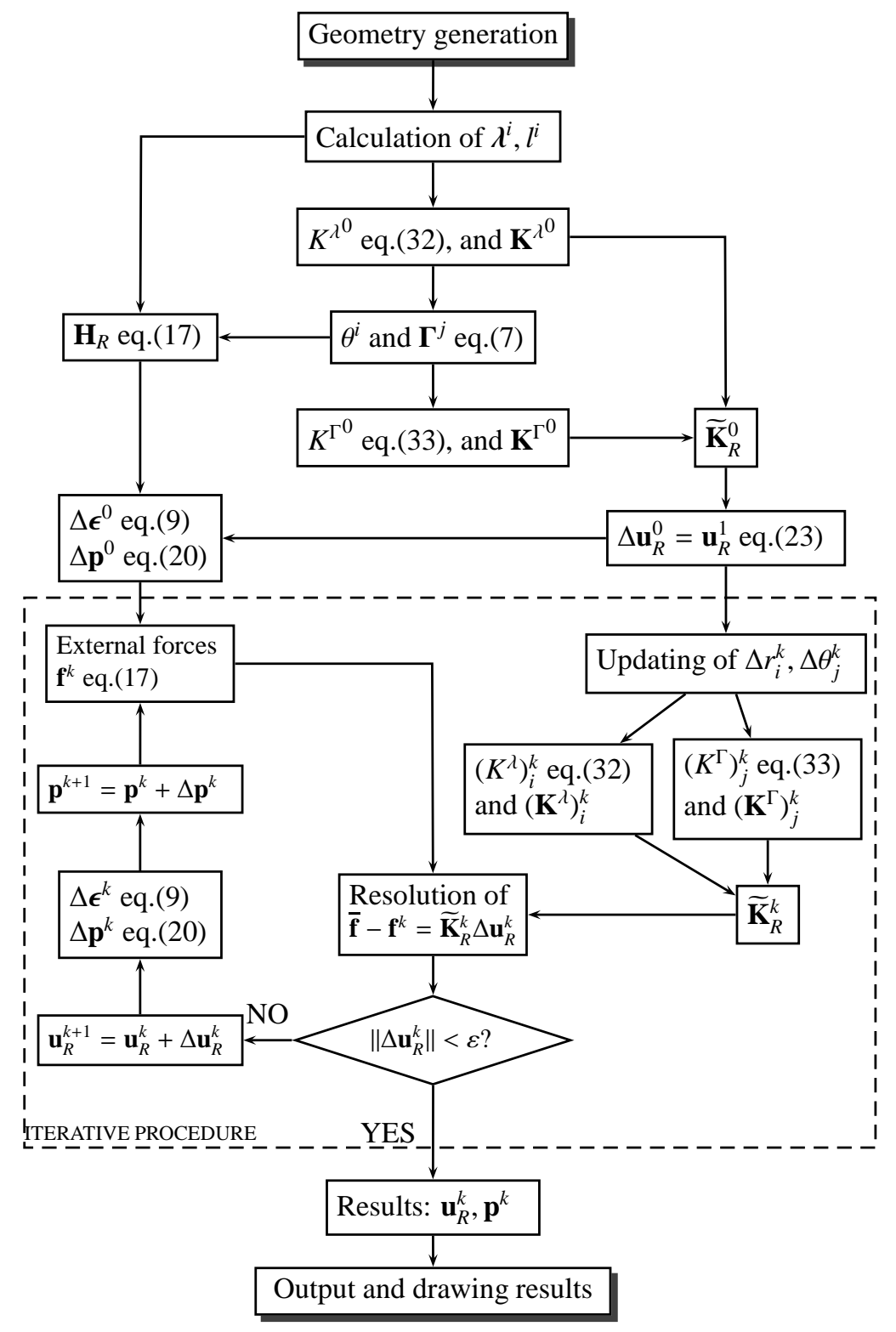

Figure 6: Iterative procedure to introduce Morse potential

\section{Numerical results and discussion}

In this section, tensile, compressive, flexural and torsional behavior of SWCNTs have been studied by using our new formulation of the 'stick-spiral' model. The following nanotubes have been tested for each loading scheme:

Similar diameters and aspect ratios have been chosen in order to further comparison of the obtained results. For the study of the mechanical properties, the usual (but controversial) wall thickness of $t=0.34 \mathrm{~nm}$ was adopted [24], [27].

One of the main issues in this paper is to find out the influence of the prestressed state and the interatomic potential in the final response. As we have taken into account two potential functions (AMBER and Morse), four calculations were carried out into each nanotube. 


\begin{tabular}{lccc} 
Chirality & $\mathrm{d}(\mathrm{nm})$ & $\mathrm{L}(\mathrm{nm})$ & $\mathrm{L} / \mathrm{d}$ \\
\hline $\mathrm{ZZ}(9,0)$ & 0.7046 & 8.5910 & 12.2 \\
$\mathrm{ZZ}(11,0)$ & 0.8612 & 10.2950 & 12.0 \\
$\mathrm{ZZ}(13,0)$ & 1.0178 & 12.2120 & 12.0 \\
\hline $\mathrm{AC}(5,5)$ & 0.6780 & 8.1164 & 12.0 \\
$\mathrm{AC}(7,7)$ & 0.9492 & 11.3138 & 11.9 \\
$\mathrm{AC}(8,8)$ & 1.0848 & 12.7895 & 11.8 \\
\hline $\mathrm{CH}(7,4)$ & 0.7541 & 8.9009 & 11.8 \\
$\mathrm{CH}(8,5)$ & 0.8879 & 9.6767 & 10.9 \\
$\mathrm{CH}(10,6)$ & 1.0960 & 11.9272 & 10.9 \\
\hline
\end{tabular}

Table 1: SWCNTs dimensions and chiralities

Cantilevered supporting conditions were assumed in all cases, following [20] and [24, fig 4], because of their easy implementation and interpretation of numerical results. Such restraints have been performed through the introduction of pinned joints at the nodes of the left end of the SWCNT, accordingly with the linear degrees of freedom (DOFs) regarded in our model.

Also, a system of point loads statically equivalent to the external loading has been applied on the atoms at the free end for each case. Of course, there is not a unique system of point loads and some additional assumption (specific for each load case) is required. A detailed description for each hypothesis and numerical values for these point loads will be given below.

\subsection{Axial behavior}

Equivalent stress-strain curves were obtained for the cantilevered SWCNTs in table 1 Stresses of 20, 40, 60, 80, $100 \mathrm{GPa}$ were tested in tension and of 30,60,120 GPa in compression.

Equivalent axial strain is calculated as:

$$
\epsilon_{x}=\frac{\Delta L}{L}
$$

where:

$\Delta L=$ variation in length of the nanotube, calculated as the longitudinal displacement at the free end.

$L=\quad$ initial length of the nanotube

Equivalent stress is evaluated as:

$$
\sigma_{x}=\frac{F_{t}}{\pi d t}
$$

where:

$F_{t}=$ total force applied in the axial direction

$d=$ nanotube diameter

$t=\quad$ thickness wall, estimated as $0.34 \mathrm{~nm}$

The point loads acting at the nodes of the right end have been obtained from $R_{i}=F_{t} / N_{t}$, where $N_{t}$ are the number of atoms along the circumferential direction of the nanotube. Hence, all the point loads into each axial simulation will be given the same values, which are provided in tables 2 and 3

For the sake of brevity, only a few $\sigma_{x}-\epsilon_{x}$ curves are plotted in fig 7 in tension and fig 8 in compression. As we can expect, linear axial behavior is reproduced with AMBER potential. Even linear behavior is yielded with Morse potential, as a direct consequence of using tangent stiffnesses into the iterative procedure. Hence, our results agree qualitatively well with those obtained from the expresions of Natsuki et. al [28] (AMBER potential) and Natsuki and Endo [30] (Morse potential) taking $t=0.34 \mathrm{~nm}$. In these references, no initial stresses were taken into account. As we can see, higher difference is observed with [30] due to the geometrical nonlinearity adopted in their work.

Despite of the controversy about mechanical properties of SWCNTs and their strong dependence on the wall thickness, the equivalent Young modulus has been calculated in each case as:

$$
E=\frac{\sigma_{x}}{\epsilon_{x}}
$$




\begin{tabular}{|c|c|c|c|c|c|c|}
\hline & \multicolumn{2}{|c|}{$R_{i}(\mathrm{nN}) F_{t}(\mathrm{nN})$} & \multicolumn{2}{|c|}{$R_{i}(\mathrm{nN}) F_{t}(\mathrm{nN})$} & \multicolumn{2}{|c|}{$R_{i}(\mathrm{nN}) F_{t}(\mathrm{nN})$} \\
\hline$\overline{\sigma_{x}(\mathrm{Gpa})}$ & \multicolumn{2}{|c|}{$\overline{Z Z}(9,0)$} & \multicolumn{2}{|c|}{$\mathrm{ZZ}(11,0)$} & \multicolumn{2}{|c|}{$\mathrm{ZZ}(13,0)$} \\
\hline 20 & 1.67 & 15.05 & 1.67 & 18.40 & 1.67 & 21.74 \\
\hline 40 & 3.34 & 30.10 & 3.34 & 36.79 & 3.34 & 43.48 \\
\hline 60 & 5.02 & 45.16 & 5.02 & 55.19 & 5.02 & 65.23 \\
\hline 80 & 6.69 & 60.21 & 6.69 & 73.59 & 6.69 & 86.97 \\
\hline 100 & 8.36 & 75.26 & 8.36 & 91.99 & 8.36 & 108.71 \\
\hline$\overline{\sigma_{x}(\mathrm{Gpa})}$ & \multicolumn{2}{|c|}{$\mathrm{AC}(5,5)$} & \multicolumn{2}{|c|}{$\mathrm{AC}(7,7)$} & \multicolumn{2}{|c|}{$\mathrm{AC}(8,8)$} \\
\hline 20 & 1.45 & 14.48 & 1.45 & 20.28 & 1.45 & 23.17 \\
\hline 40 & 2.90 & 28.97 & 2.90 & 40.56 & 2.90 & 46.35 \\
\hline 60 & 4.35 & 43.45 & 4.35 & 60.83 & 4.35 & 69.52 \\
\hline 80 & 5.79 & 57.94 & 5.79 & 81.11 & 5.79 & 92.70 \\
\hline 100 & 7.24 & 72.42 & 7.24 & 101.39 & 7.24 & 115.87 \\
\hline$\overline{\sigma_{x}(\mathrm{Gpa})}$ & \multicolumn{2}{|c|}{$\mathrm{CH}(7,4)$} & \multicolumn{2}{|c|}{$\mathrm{CH}(8,5)$} & \multicolumn{2}{|c|}{$\mathrm{CH}(10,6)$} \\
\hline 20 & 1.46 & 16.11 & 1.46 & 18.97 & 1.46 & 23.41 \\
\hline 40 & 2.93 & 32.22 & 2.92 & 37.93 & 2.93 & 46.83 \\
\hline 60 & 4.39 & 48.33 & 4.38 & 56.90 & 4.39 & 70.24 \\
\hline 80 & 5.86 & 64.44 & 5.84 & 75.87 & 5.85 & 93.66 \\
\hline 100 & 7.32 & 80.55 & 7.30 & 94.84 & 7.32 & 117.07 \\
\hline
\end{tabular}

Table 2: Point loads applied in tensile simulations

\begin{tabular}{|c|c|c|c|}
\hline & $R_{i}(\mathrm{nN}) F_{t}(\mathrm{nN})$ & $R_{i}(\mathrm{nN}) F_{t}(\mathrm{nN})$ & $R_{i}(\mathrm{nN}) F_{t}(\mathrm{nN})$ \\
\hline$\overline{\sigma_{x}(\mathrm{Gpa})}$ & $\mathrm{ZZ}(9,0)$ & $\mathrm{ZZ}(11,0)$ & $\mathrm{ZZ}(13,0)$ \\
\hline 30 & \begin{tabular}{|ll}
2.51 & 22.58 \\
\end{tabular} & $\begin{array}{ll}2.51 & 27.60\end{array}$ & $\begin{array}{ll}2.51 & 32.61\end{array}$ \\
\hline 60 & $5.02 \quad 45.16$ & $5.02 \quad 55.19$ & $5.02 \quad 65.23$ \\
\hline 120 & $10.03 \quad 90.31$ & $10.03 \quad 110.38$ & $10.03 \quad 130.45$ \\
\hline$\overline{\sigma_{x}(\mathrm{Gpa})}$ & $\mathrm{AC}(5,5)$ & $\mathrm{AC}(7,7)$ & $\mathrm{AC}(8,8)$ \\
\hline 30 & \begin{tabular}{|l|}
2.17 \\
21.73
\end{tabular} & 2.1730 .42 & 2.1734 .76 \\
\hline 60 & $4.35 \quad 43.45$ & $4.35 \quad 60.83$ & $4.35 \quad 69.52$ \\
\hline 120 & $8.69 \quad 86.90$ & $8.69 \quad 121.67$ & $8.69 \quad 139.05$ \\
\hline$\sigma_{x}(\mathrm{Gpa})$ & $\mathrm{CH}(7,4)$ & $\mathrm{CH}(8,5)$ & $\mathrm{CH}(10,6)$ \\
\hline 30 & \begin{tabular}{|ll}
2.20 & 24.17
\end{tabular} & \begin{tabular}{|ll}
2.19 & 28.45
\end{tabular} & $\begin{array}{ll}2.20 & 35.12\end{array}$ \\
\hline 60 & $4.39 \quad 48.33$ & $4.38 \quad 56.90$ & $4.39 \quad 70.24$ \\
\hline 120 & $8.79 \quad 96.66$ & $8.75 \quad 113.80$ & $8.78 \quad 140.49$ \\
\hline
\end{tabular}

Table 3: Point loads applied in compressive simulations

averaging results in each curve. Thus, we can analyse the relationship among the nanotube diameter and its Young modulus. Final results in tension are summarized in table 4 and plotted in figure 9.

From figures 7 and 9 we can state that axial response in tension is stiffer (on average terms) by using Morse potential than AMBER potential. Otherwise, AMBER and Morse potential agree reasonably well in tension for axial strains below $6 \%$ (see fig77).

In general, Young modulus grows slightly with nanotube diameter if prestresses are not included, in agreement with other published works ([26, fig 4a], [31], [27], [24, fig 12], [21, fig 7]). Nevertheless, the opposite trend is observed if prestressed state is regarded. In fact, including the prestressed state increases longitudinal stiffness up to $18 \%$ except for $\mathrm{ZZ}(9,0)$, where a stiffening of $32 \%$ is obtained. This remarkable effect may be explained as follows: initial stresses introduce radial resultant forces which trend to keep the circular cross-section of the nanotube and therefore produce a longitudinal shortening. Obviously the lower loads are applied, the higher stiffening effect of the prestressed state in tension is rendered. 


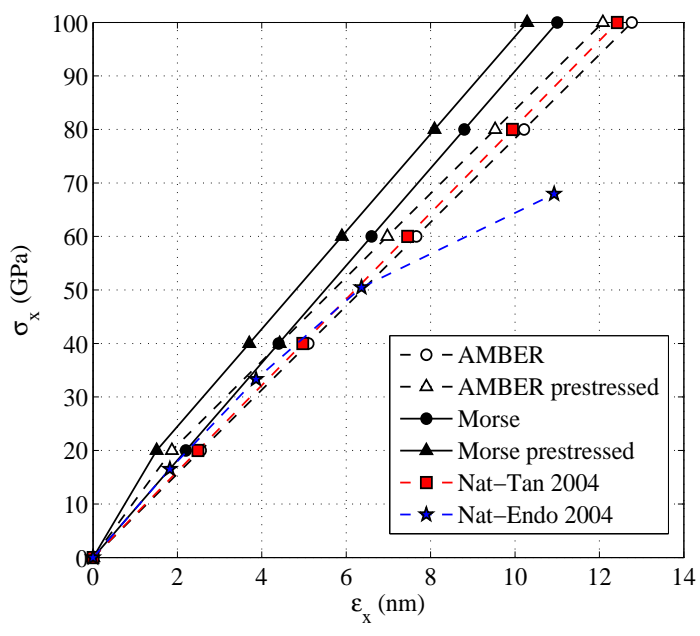

(a) $\mathrm{ZZ}(11,0)$ tension

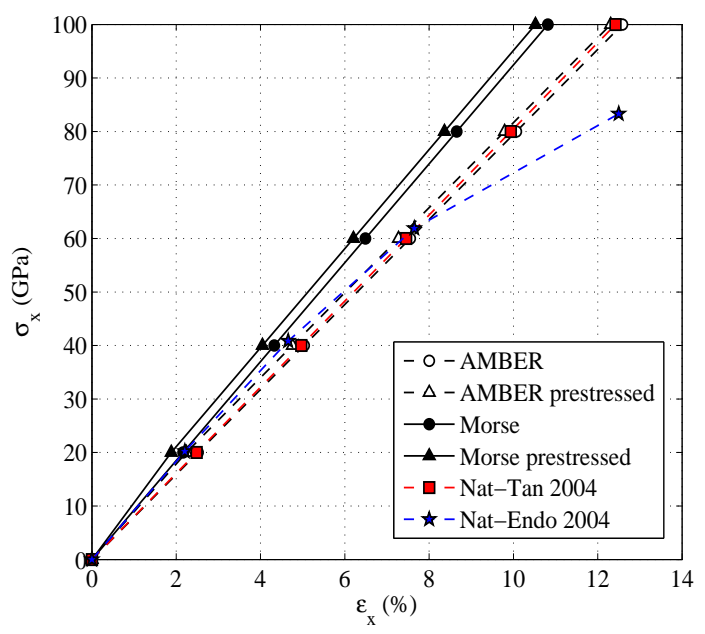

(b) $\mathrm{AC}(7,7)$ tension

Figure 7: SWCNTs tensile behavior

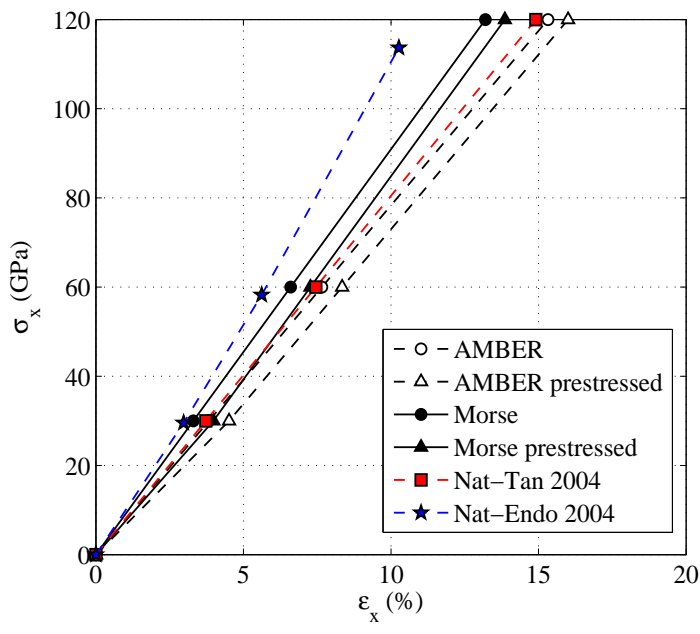

(a) $\mathrm{ZZ}(11,0)$ compression

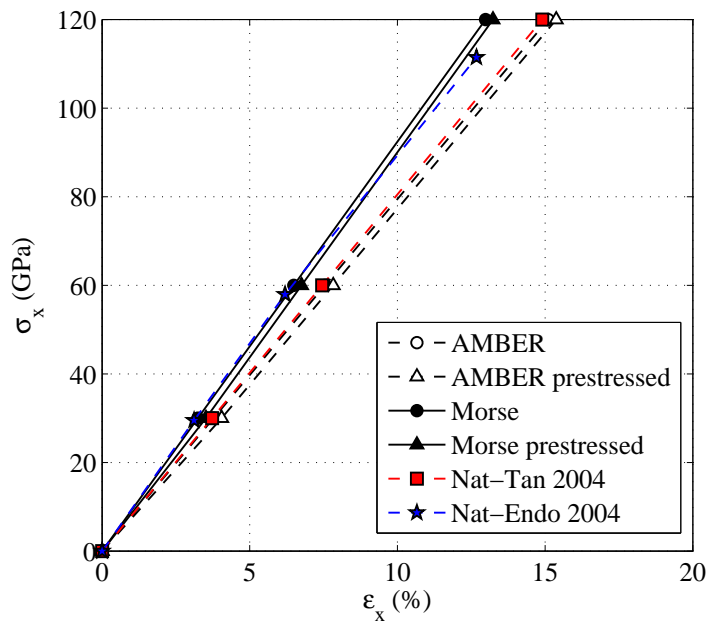

(b) $\mathrm{AC}(7,7)$ compression

Figure 8: SWCNTs compressive behavior

Tensile simulations with Chiral CNTs diverge for high loads due to the non-axisymmetry of this nanotubes: Chiral tensile loaded CNTs involve some bonds strongly tensioned and some other bonds strongly compressed, therefore, the very different stiffnesses from tension to compression with Morse potential (fig 4(a) causes ill-conditioning of the iterative procedure depicted in figure 6. Further improvements of this numerical procedure are currently in progress.

Other disadvantages of the non-axisymmetry of Chiral CNTs are the coupling between tension and torsion, and the local transversal deformation at the free end (see figure $10(\mathrm{c})$ ) which can distort the final results. This deformation can be avoided by imposing displacements at the right end of the CNT in order to keep the ending atoms into the CNT circular cross-section. These new boundary conditions will be included in future works.

Our results agree reasonably well with those reported by [30], [20] and [24] for nanotubes of the same diameter. 


\begin{tabular}{lrrrrr}
\cline { 3 - 6 } & & \multicolumn{3}{c}{ AMBER AMBER MORSE MORSE } \\
\cline { 3 - 6 } & $\mathrm{d}(\mathrm{nm})$ & $\mathrm{E}(\mathrm{GPa})$ & $\mathrm{E}(\mathrm{GPa})$ & $\mathrm{E}(\mathrm{GPa})$ & $\mathrm{E}(\mathrm{GPa})$ \\
\hline $\mathrm{ZZ}(9,0)$ & 0.7046 & 774.46 & 967.75 & 897.63 & 1184.68 \\
$\mathrm{ZZ}(11,0)$ & 0.8612 & 783.24 & 900.27 & 909.09 & 1076.76 \\
$\mathrm{ZZ}(13,0)$ & 1.0178 & 788.67 & 868.55 & 916.19 & 1029.00 \\
\hline $\mathrm{AC}(5,5)$ & 0.6780 & 786.54 & 873.54 & 913.83 & 1038.90 \\
$\mathrm{AC}(7,7)$ & 0.9492 & 794.54 & 837.41 & 924.39 & 984.89 \\
$\mathrm{AC}(8,8)$ & 1.0848 & 796.77 & 829.27 & 927.11 & 972.96 \\
\hline $\mathrm{CH}(7,4)$ & 0.7541 & 778.97 & 845.14 & 905.94 & 1040.18 \\
$\mathrm{CH}(8,5)$ & 0.8879 & 783.96 & 829.12 & 900.02 & 1018.43 \\
$\mathrm{CH}(10,6)$ & 1.0960 & 784.06 & 814.20 & 904.24 & 982.74 \\
\hline
\end{tabular}

Table 4: Young modulus SWCNTs in tension

In order to derive the chirality influence on the tensile behavior, two more SWCNTs under tension by using AMBER potential with initial stresses (table 5) have been tested and compared with the SWCNTs from table 1 with the intermediate diameter in each Chirality. Results are summarized in table 6 and plotted in figure 11

\begin{tabular}{lccc} 
Chirality & $\mathrm{d}(\mathrm{nm})$ & $\mathrm{L}(\mathrm{nm})$ & $\mathrm{L} / \mathrm{d}$ \\
\hline $\mathrm{CH}(8,4)$ & 0.8285 & 10.1436 & 12.2 \\
$\mathrm{CH}(10,4)$ & 0.9707 & 11.5281 & 11.9 \\
\hline
\end{tabular}

Table 5: Additional SWCNTs dimensions and chiralities

\begin{tabular}{|c|c|c|c|c|c|}
\hline & $\mathrm{d}(\mathrm{nm})$ & $\mathrm{L}(\mathrm{nm})$ & $\mathrm{L} / \mathrm{d}$ & $\theta\left(^{\circ}\right)$ & $\mathrm{E}(\mathrm{GPa})$ \\
\hline $\mathrm{ZZ}(11,0)$ & 0.8612 & 10.2950 & 12.0 & 0.0 & 900.27 \\
\hline $\mathrm{CH}(10,4)$ & 0.9707 & 11.5281 & 11.9 & 16.1 & 834.87 \\
\hline $\mathrm{CH}(8,4)$ & 0.8285 & 10.1436 & 12.2 & 19.1 & 842.32 \\
\hline $\mathrm{CH}(8,5)$ & 0.8879 & 9.6767 & 10.9 & 22.4 & 829.12 \\
\hline $\mathrm{AC}(7,7)$ & 0.9492 & 11.3138 & 11.9 & 30.0 & 837.41 \\
\hline
\end{tabular}

Table 6: Young Modulus (tension) against Chirality

On the whole, ZigZag CNTs appear to be about $8 \%$ stiffer than Armchair ones, against [30]. Nevertheless, the diameter influence on the Young modulus can not be removed from the values in table 6 and there is not a clear trend in the figure 11

Let us draw our attention on the compressive behavior of SWNCTs. As can be seen from figure 8 , Morse potential produces stiffer results in compression than AMBER potential because of the higher slope in the compressive branch for bond elongation of the former. In fact, the difference is clear from axial strains of $6 \%$.

Proceeding as we did in tension, we can obtain new values for the Young modulus in compression (table 7) and plot them against nanotube diameter (see figure 12). In this case, Young modulus is increasing with the nanotube diameter, but in the range of diameters studied herein we could consider it nearly constant.

Regarding initial stresses, their effect is the opposite we found in tension. Therefore, the shortening caused by the prestressed state decreases the compressive Young modulus (figure 12).

Obviously, if Morse potential or prestressed state are involved in the analysis, different Young moduli are obtained from tension to compression and the applicability of continuum models becomes doubtful, as has been remarked in previous published works [27], [26]. 


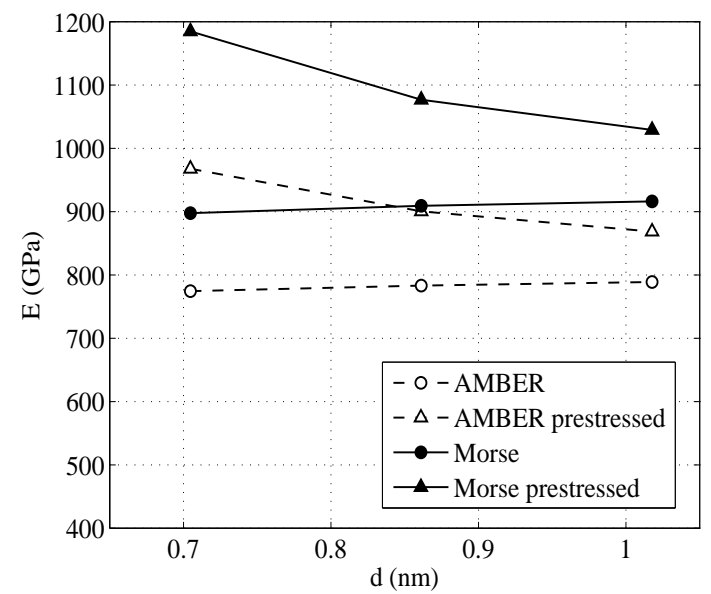

(a) ZigZag SWCNTs tension

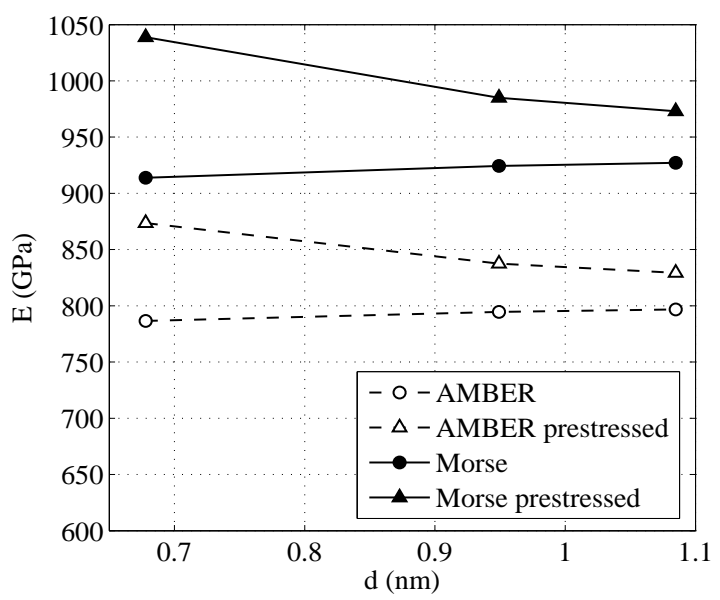

(b) Armchair SWCNTs tension

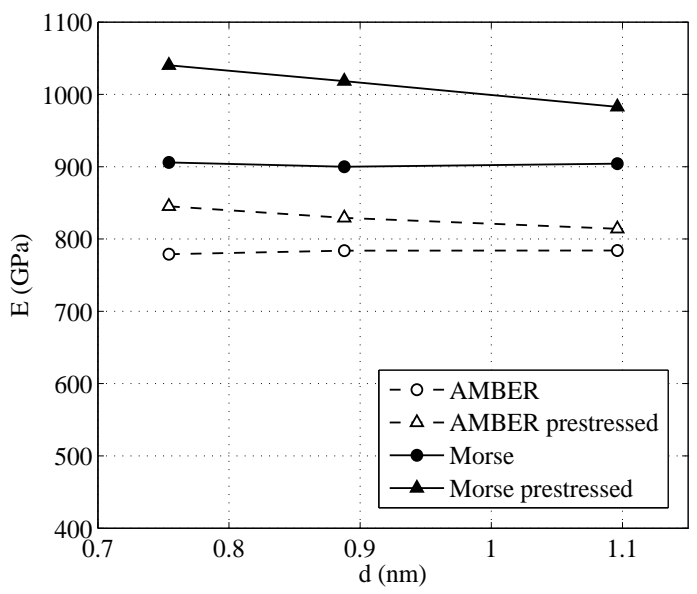

(c) Chiral SWCNTs tension

Figure 9: Young modulus SWCNTs in tension

The ill-conditioning of the iterative procedure is again present for Chiral SWCNTs with Morse potential at high load levels. Likewise, the local transverse deformation at the free end can be observed in figure 13(c),

From table 7, chirality does not have a relevant effect on the compressive response and it could be readily neglected as we concluded in tension.

\subsection{Flexural behavior}

Cantilever supporting conditions (through pinned joints) were kept for flexural loading and a external bending moment was applied at the free end, taking values from table 8

Each bending moment has been coverted into a system of statically equivalent longitudinal point loads at the nodes of the free end. If we define the $z$-axis as the bending axis and $y$ as the orthogonal coordinate, the values of the point loads will vary in linear proportion with the $y$ coordinate, following:

$$
R_{x i}=\frac{M z}{\sum y_{i}^{2}} y_{i}
$$



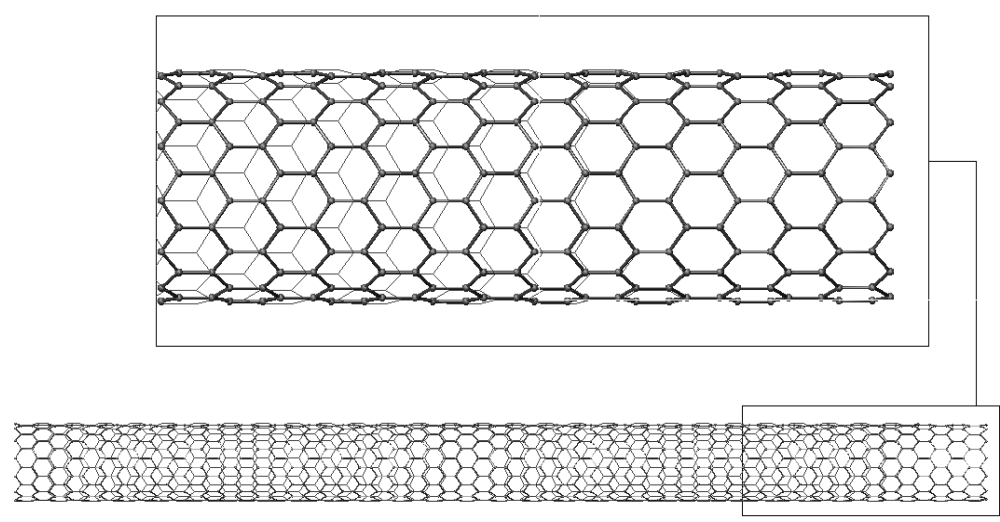

(a) $\operatorname{ZigZag}(13,0) \sigma_{x}=60 G P a$, AMBER

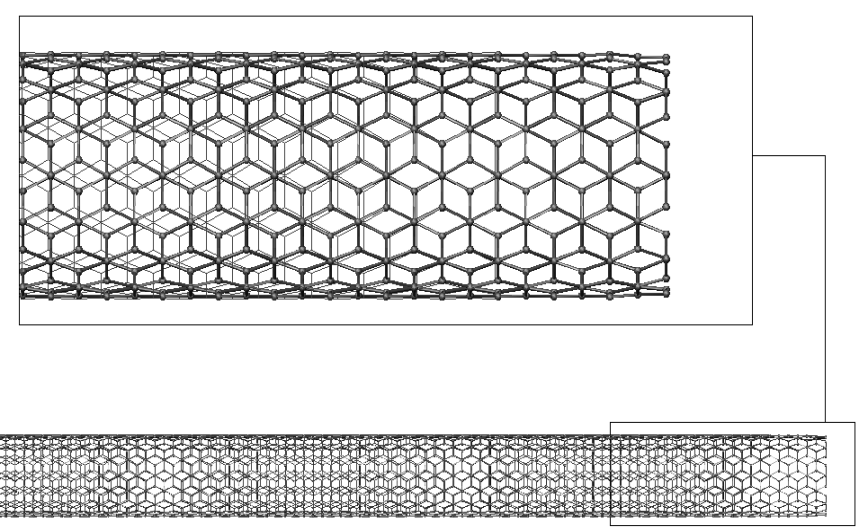

(b) $\operatorname{Armchair}(8,8) \sigma_{x}=60 G P a$, MORSE prestressed

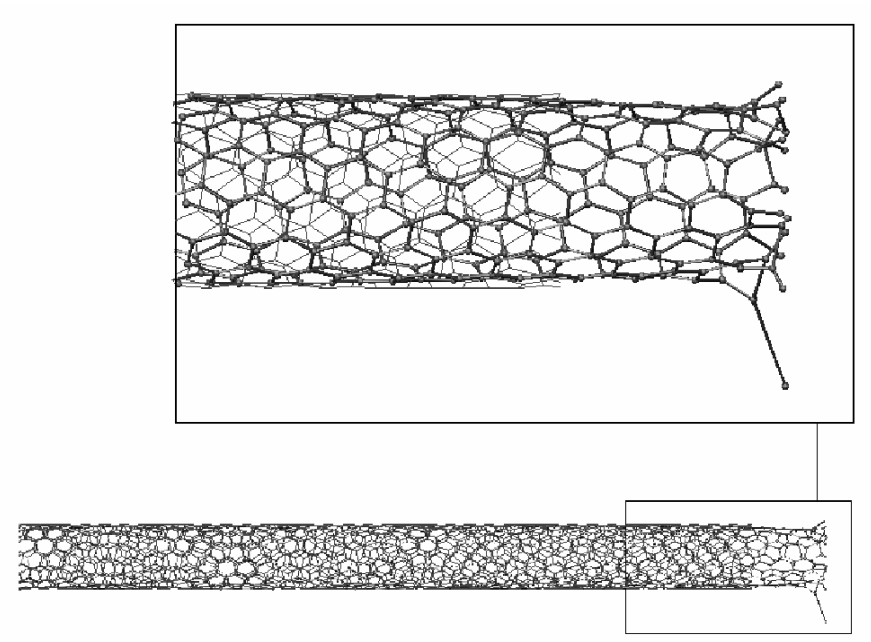

(c) $\operatorname{Chiral}(8,5) \sigma_{x}=80 G P a$, AMBER

Figure 10: Deformed shape SWCNTs tension 


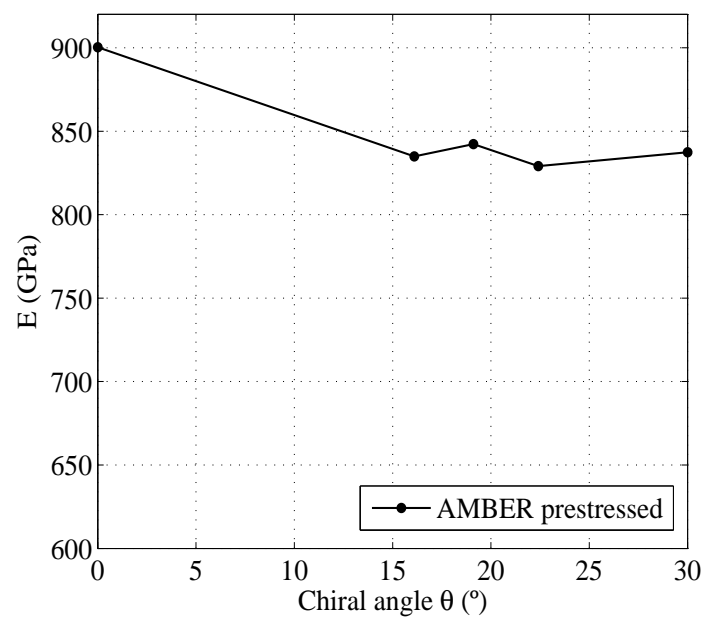

Figure 11: Influence of the Chirality on the Young Modulus (tension)

\begin{tabular}{lllccc}
\cline { 3 - 6 } & & \multicolumn{3}{c}{ AMBER AMBER MORSE MORSE } \\
\cline { 3 - 6 } & $\mathrm{d}(\mathrm{nm})$ & $\mathrm{E}(\mathrm{GPa})$ & $\mathrm{E}(\mathrm{GPa})$ & $\mathrm{E}(\mathrm{GPa})$ & $\mathrm{E}(\mathrm{GPa})$ \\
\hline $\mathrm{ZZ}(9,0)$ & 0.7046 & 774.41 & 674.55 & 897.52 & 768.28 \\
$\mathrm{ZZ}(11,0)$ & 0.8612 & 783.21 & 711.09 & 909.07 & 814.99 \\
$\mathrm{ZZ}(13,0)$ & 1.0178 & 788.68 & 734.57 & 916.24 & 845.27 \\
\hline $\mathrm{AC}(5,5)$ & 0.6780 & 786.48 & 728.64 & 913.81 & 839.61 \\
$\mathrm{AC}(7,7)$ & 0.9492 & 794.66 & 762.57 & 924.39 & 882.88 \\
$\mathrm{AC}(8,8)$ & 1.0848 & 796.78 & 771.68 & 927.13 & 894.61 \\
\hline $\mathrm{CH}(7,4)$ & 0.7541 & 795.56 & 718.80 & 905.87 & 814.66 \\
$\mathrm{CH}(8,5)$ & 0.8879 & 780.97 & 741.93 & 900.15 & 847.50 \\
$\mathrm{CH}(10,6)$ & 1.0960 & 799.16 & 761.45 & 904.26 & 882.50 \\
\hline
\end{tabular}

Table 7: Young modulus SWCNTs in compression

\begin{tabular}{cccc}
\cline { 2 - 4 } & $\mathrm{ZZ}(9,0)$ & $\mathrm{ZZ}(11,0)$ & $\mathrm{ZZ}(13,0)$ \\
& $\mathrm{AC}(5,5)$ & $\mathrm{AC}(7,7)$ & $\mathrm{AC}(8,8)$ \\
& $\mathrm{CH}(7,4)$ & $\mathrm{CH}(8,5)$ & $\mathrm{CH}(10,6)$ \\
\hline \multirow{3}{*}{$\mathrm{M}(n N \cdot n m)$} & 0.5 & 1.0 & 1.0 \\
& 1.0 & 2.0 & 2.0 \\
& 2.0 & 3.0 & 4.0 \\
\hline
\end{tabular}

Table 8: Bending moments $(n N \cdot n m)$ applied at the free end

Some numerical values of the applied nodal forces under a bending moment of $M=1 \mathrm{nN} \cdot \mathrm{nm}$ are included in table 9 . where positive values indicate tensile forces and negative values stand for compressive forces.

Aimed to find out qualitatively the bending response of SWCNTs, rotations at the free end have been measured and plotted against bending moment. All curves plotted show nearly linear flexural behavior (see figure 14 as an example), even when Morse potential is taken into account. Therefore, nonlinear bond interaction of the potential function has a little influence on the bending response, as could be expected. Nevertheless, the choice of the potential 


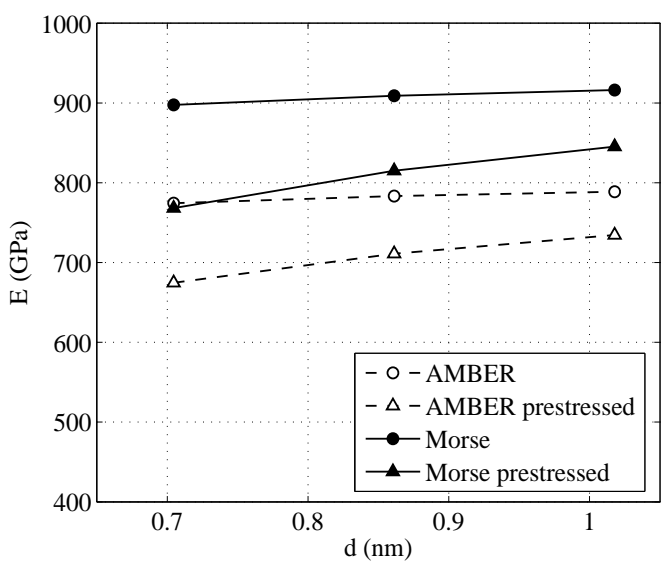

(a) ZigZag SWCNTs compression

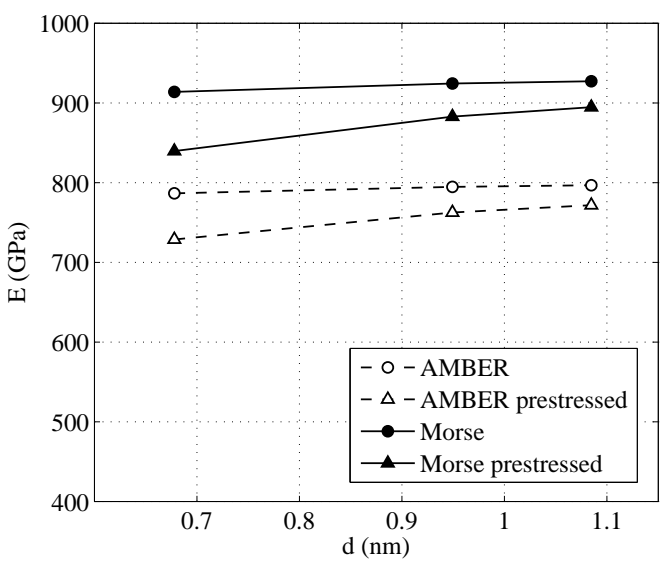

(b) Armchair SWCNTs compression

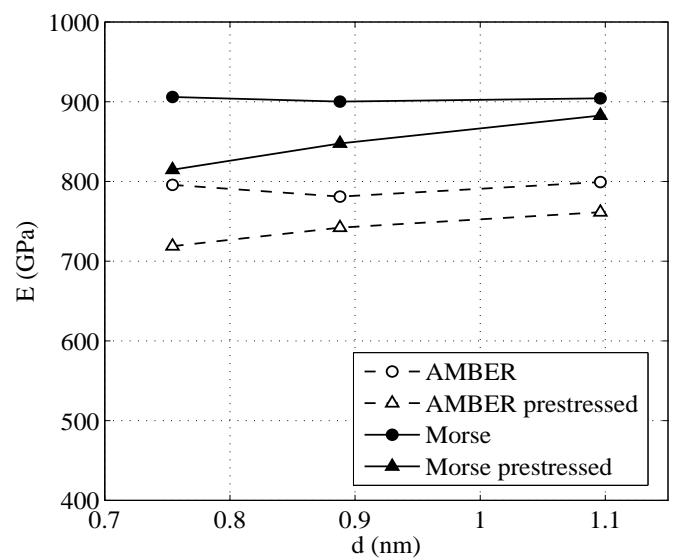

(c) Chiral SWCNTs compression

Figure 12: Young modulus SWCNTs in compression

has more influence than initial stresses, which could be readily neglected. As well, the Young modulus in tension derived from [28] ( $E=805^{\prime} 01 \mathrm{GPa}$ ) is used to plot additional bending curves in figs 14(a) and 14(b) respectively, giving stiffer results than the equivalent curve with our model in around $23 \%$.

In order to compare mechanical parameters obtained from axial behavior with those from bending response, Young moduli were calculated in this load case as:

$$
E=\frac{M L}{I \theta}
$$

where:

$M=$ moment at the free end

$\theta=$ rotation at the free end

$L=\quad$ initial length of the SWCNT

$I=$ moment of inertia of the cross-section regarding the SWCNT as a hollow cylinder with $t=0.34 \mathrm{~nm}$

Averaging values for each nanotube we can obtain the values of table 10 . This results show a substantial scattering of the Young modulus and stress the fact that continuum models (and particularly standard beam theories) are not applicable at that nano-scale size due to the relatively small amount of atoms involved. This idea is in clear disagreement with other published works (e.g. [6], [10], [11], [13], [14], [16]). 


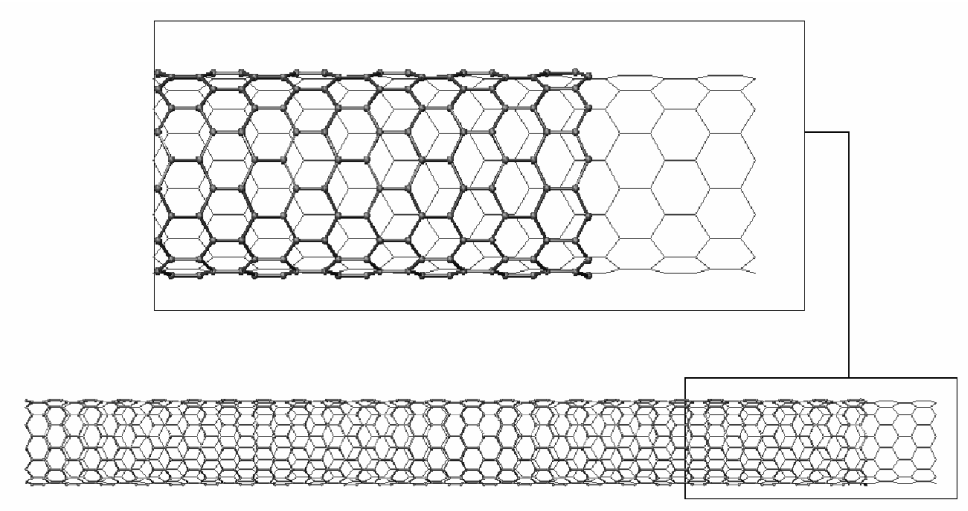

(a) ZigZag $(11,0) \sigma_{x}=60 G P a$, AMBER

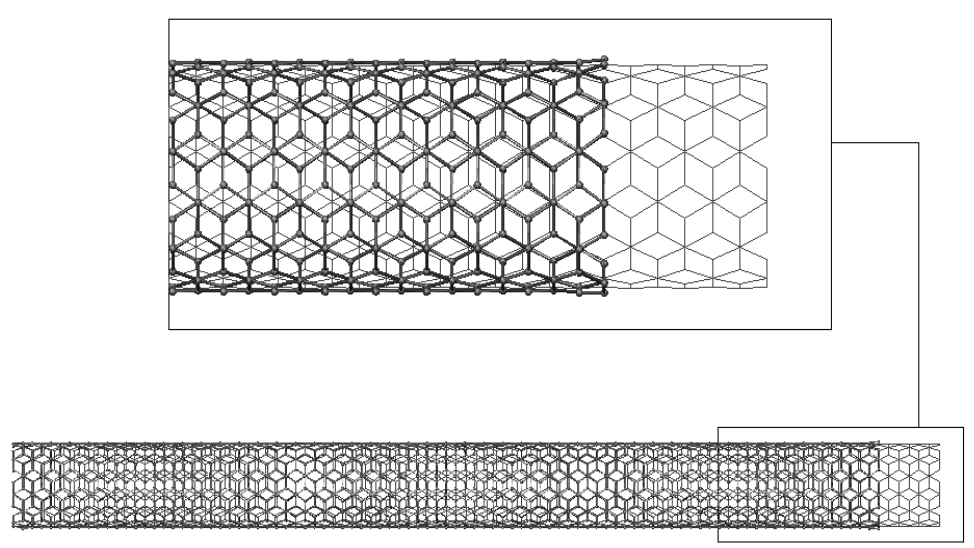

(b) $\operatorname{Armchair}(7,7) \sigma_{x}=60 G P a$, MORSE

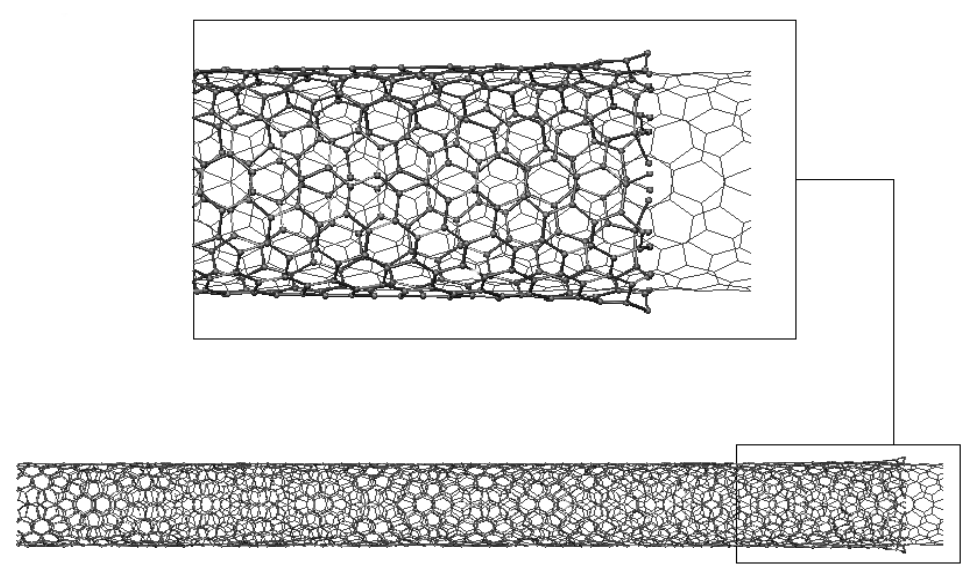

(c) $\operatorname{Chiral}(10,6) \sigma_{x}=30 G P a$, AMBER prestressed

Figure 13: Deformed shape SWCNTs compression

Then, Young modulus obtained by eq. (40) is plotted in figure 15. As we can see, Young modulus is increasing (as in tension) with diameter for ZigZag and Armchair nanotubes, but surprisingly is decreasing with diameter with Chiral nanotubes. It should be noted that numerical ill-conditioning is particularly important with small diameter 


\begin{tabular}{ccccccc}
\multicolumn{2}{c}{$\mathrm{ZZ}(11,0)$} & \multicolumn{2}{c}{$\mathrm{AC}(5,5)$} & \multicolumn{2}{c}{$\mathrm{CH}(7,4)$} \\
\hline$y_{i}(\mathrm{~nm})$ & $R_{x i}(\mathrm{nN})$ & \multicolumn{2}{c}{$y_{i}(\mathrm{~nm})$} & $R_{x i}(\mathrm{nN})$ & $y_{i}(\mathrm{~nm})$ & $R_{x i}(\mathrm{nN})$ \\
\hline 0.4131 & 0.41 & 0.3390 & 0.59 & 0.3693 & 0.47 \\
0.4131 & 0.41 & 0.3097 & 0.54 & 0.3547 & 0.45 \\
0.2820 & 0.28 & 0.2268 & 0.39 & 0.2892 & 0.37 \\
0.2820 & 0.28 & 0.1048 & 0.18 & 0.1888 & 0.24 \\
0.0613 & 0.06 & 0.1048 & 0.18 & 0.0655 & 0.08 \\
0.0613 & 0.06 & -0.0354 & -0.06 & 0.0394 & 0.05 \\
-0.1789 & -0.18 & -0.1354 & -0.29 & -0.0117 & -0.15 \\
-0.1789 & -0.18 & -0.2743 & -0.48 & -0.1887 & -0.24 \\
-0.3622 & -0.36 & -0.2743 & -0.48 & -0.2892 & -0.37 \\
-0.3622 & -0.36 & -0.3316 & -0.58 & -0.3449 & -0.44 \\
-0.4306 & -0.42 & & & -0.3775 & -0.48 \\
\hline
\end{tabular}

Table 9: Point loads applied in bending for $M=1 n N \cdot n m$

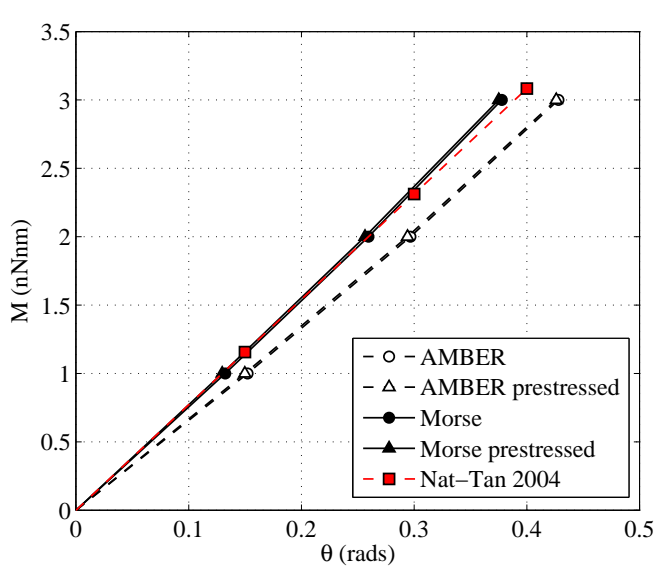

(a) ZZ(11,0) bending

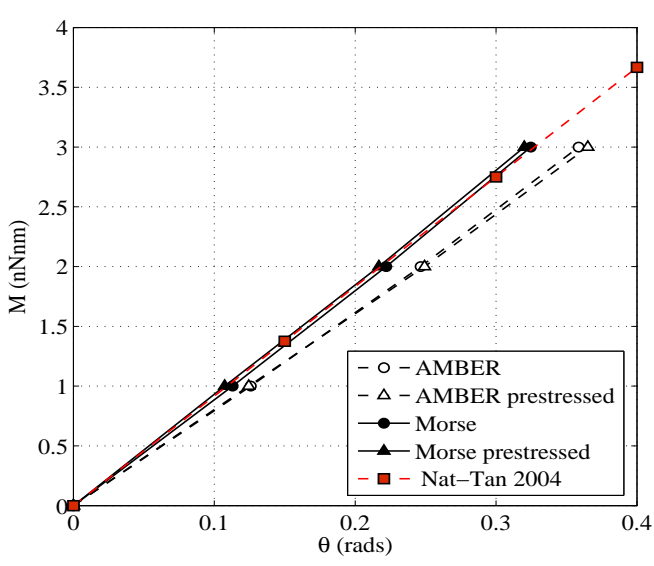

(b) $\mathrm{AC}(7,7)$ bending

Figure 14: SWCNTs flexural behavior

\begin{tabular}{llllll}
\cline { 3 - 6 } & & \multicolumn{4}{c}{ AMBER AMBER MORSE MORSE } \\
& prestress & prestress \\
\cline { 3 - 6 } & $\mathrm{d}(\mathrm{nm}(\mathrm{GPa})$ & $\mathrm{E}(\mathrm{GPa})$ & $\mathrm{E}(\mathrm{GPa})$ & $\mathrm{E}(\mathrm{GPa})$ \\
\hline $\mathrm{ZZ}(9,0)$ & 0.7046 & 652.25 & 661.96 & 742.78 & 758.32 \\
$\mathrm{ZZ}(11,0)$ & 0.8612 & 707.34 & 714.23 & 807.76 & 818.36 \\
$\mathrm{ZZ}(13,0)$ & 1.0178 & 733.35 & 741.34 & 841.04 & 853.42 \\
\hline $\mathrm{AC}(5,5)$ & 0.6780 & 655.01 & 652.39 & 716.82 & 749.63 \\
$\mathrm{AC}(7,7)$ & 0.9492 & 714.88 & 710.40 & 793.20 & 816.92 \\
$\mathrm{AC}(8,8)$ & 1.0848 & 709.03 & 727.62 & 811.29 & 837.80 \\
\hline $\mathrm{CH}(7,4)$ & 0.7541 & 758.04 & 789.15 & 893.94 & 689.50 \\
$\mathrm{CH}(8,5)$ & 0.8879 & 614.42 & 721.82 & 782.10 & 839.13 \\
$\mathrm{CH}(10,6)$ & 1.0960 & 607.76 & 710.09 & 781.07 & 821.92 \\
\hline
\end{tabular}

Table 10: Young modulus SWCNTs in bending 
Chiral SWCNTs in bending and could be the reason for this opposite trend.

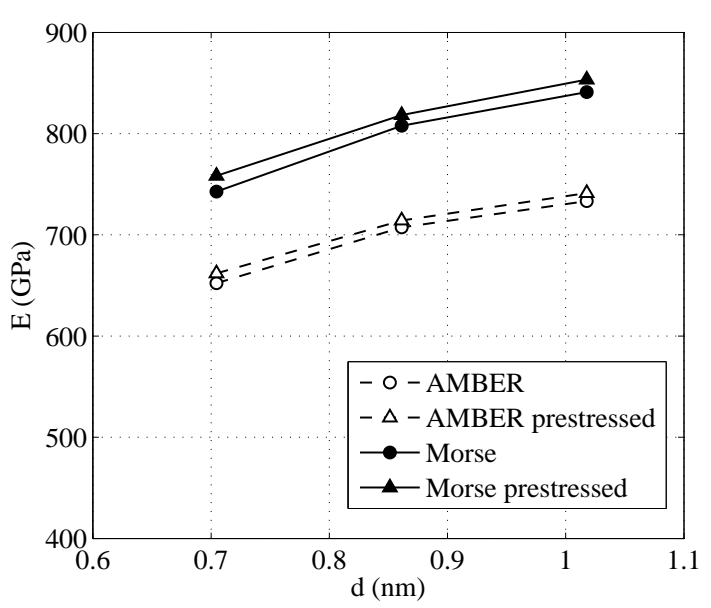

(a) ZigZag SWCNTs bending

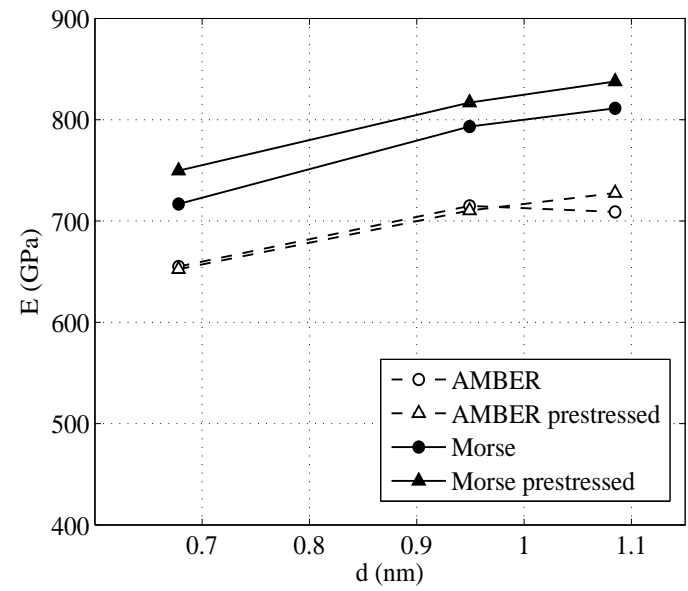

(b) Armchair SWCNTs bending

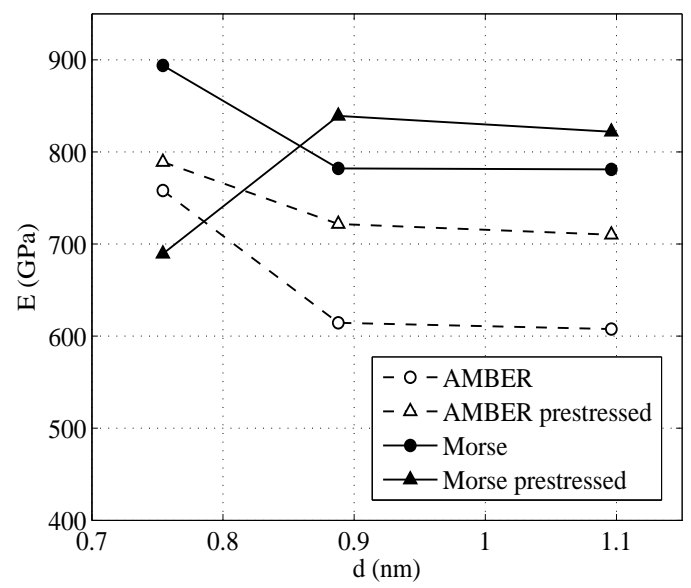

(c) Chiral SWCNTs bending

Figure 15: Young modulus SWCNTs in bending

Comparing values of table 10, the influence of the chirality in the flexural behavior may be omitted, since there is not a clear difference in Young modulus between ZigZag and Armchair nanotubes. Then, bending of SWCNTs (as tension and compression) is nearly independent of the chirality. Qualitative bending response of SWCNTs may be observed in figure 16

\subsection{Torsional behavior}

Finally, cantilevered SWCNTs (pinned joints) were subjected to a set of point loads $F_{t i}$ at their free end producing a torque with the same values taken in bending (see table 8). These loads are assumed to be tangent to the extreme circumference of the tube at the free end, and all of them should produce the same torque with respect to the cylinder axis. Hence, they can be defined by

$$
F_{t i}=\frac{2 M_{t}}{N_{t} d}
$$




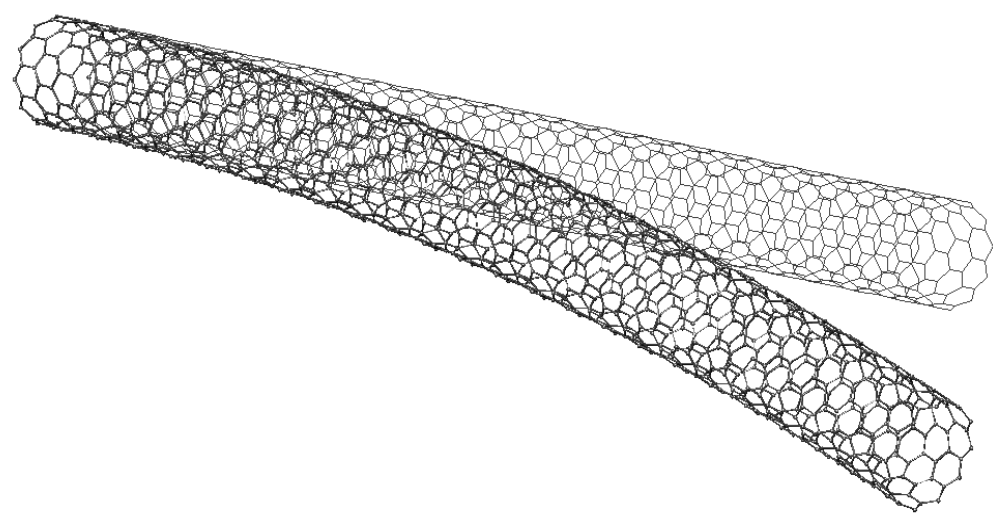

(a) ZigZag(11,0) $M=2 n N n m$, AMBER prestressed

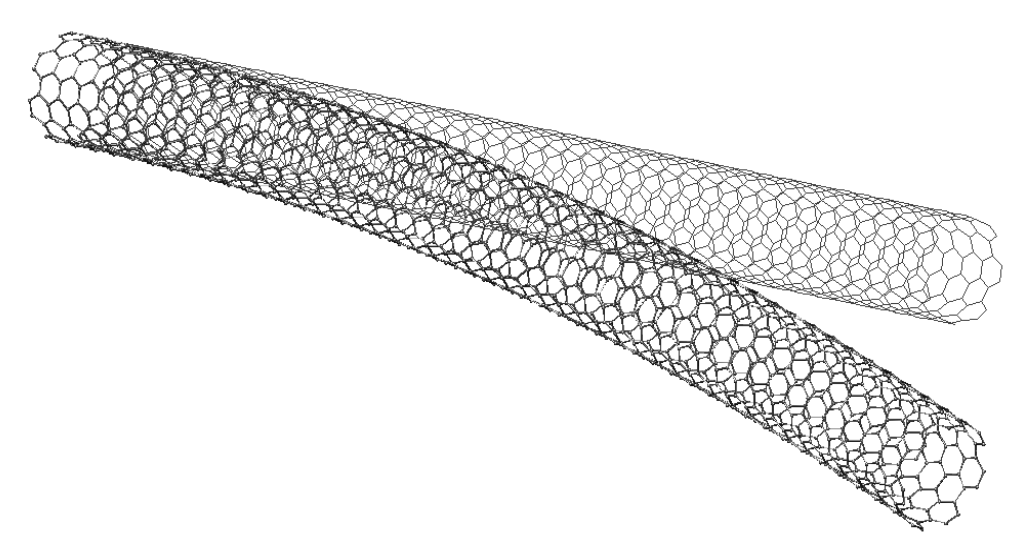

(b) Armchair(7,7) $M=3 n N n m$, MORSE prestressed

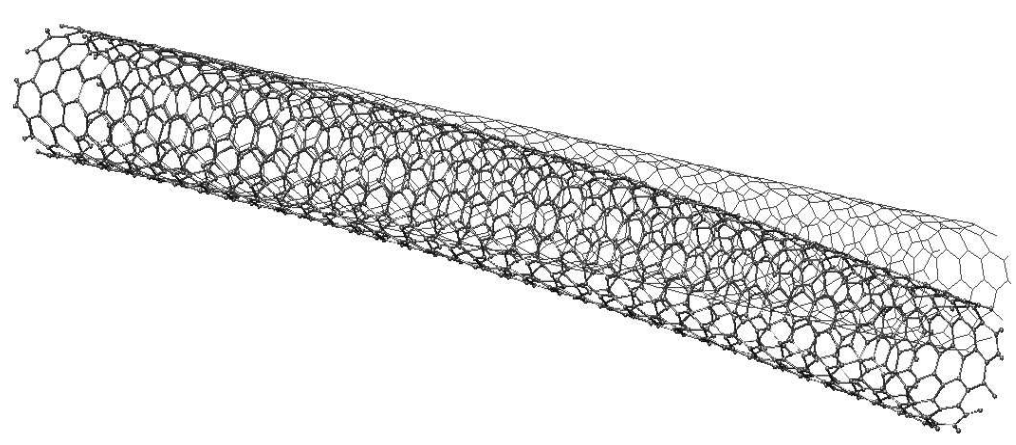

(c) $\operatorname{Chiral}(8,5) M=1 n N n m$, MORSE prestressed

Figure 16: Deformed shape SWCNTs bending

and conveniently projected onto the global axes. Some numerical values for the final components of these forces taking a torque of $M_{t}=1 n \cdot \mathrm{Nnm}$ are given in table 11 .

Into each simulation, rotation of the free end is measured and plotted against torsional moment (see figure 17 as an example). Our results are compared with those obtained from [28] by using AMBER potential. The difference in about 35\% may be because the approach in [28] is related to the planar graphene sheet without taking into account 


\begin{tabular}{cccccccccccc}
\multicolumn{1}{c}{$\mathrm{ZZ}(11,0)$} & \multicolumn{1}{c}{$\mathrm{AC}(5,5)$} & \multicolumn{4}{c}{$\mathrm{CH}(7,4)$} \\
\hline$y_{i}(\mathrm{~nm})$ & $z_{i}(\mathrm{~nm})$ & $R_{y i}(\mathrm{nN})$ & $R_{z i}(\mathrm{nN})$ & $y_{i}(\mathrm{~nm})$ & $z_{i}(\mathrm{~nm})$ & $R_{y i}(\mathrm{nN})$ & $R_{z i}(\mathrm{nN})$ & $y_{i}(\mathrm{~nm})$ & $z_{i}(\mathrm{~nm})$ & $R_{y i}(\mathrm{nN}) R_{z i}(\mathrm{nN})$ \\
\hline 0.0613 & -0.4262 & 0.21 & 0.03 & 0.1048 & -0.3224 & 0.28 & 0.09 & 0.0394 & -0.3754 & 0.24 & 0.03 \\
0.2820 & -0.3254 & 0.16 & 0.14 & 0.2268 & -0.2520 & 0.22 & 0.20 & 0.1888 & -0.3269 & 0.21 & 0.12 \\
0.4131 & -0.1213 & 0.06 & 0.20 & 0.3390 & 0.0000 & 0.00 & 0.29 & 0.3547 & -0.1291 & 0.08 & 0.23 \\
0.4131 & 0.1213 & -0.06 & 0.20 & 0.3097 & 0.1379 & -0.12 & 0.27 & 0.3693 & 0.0784 & -0.05 & 0.24 \\
0.2820 & 0.3254 & -0.16 & 0.14 & 0.1048 & 0.3224 & -0.28 & 0.09 & 0.2892 & 0.2427 & -0.15 & 0.18 \\
0.0613 & 0.4262 & -0.21 & 0.03 & -0.0354 & 0.3371 & -0.27 & -0.03 & 0.0655 & 0.3718 & -0.24 & 0.04 \\
-0.1789 & 0.3917 & -0.19 & -0.09 & -0.2743 & 0.1993 & -0.17 & -0.24 & -0.1166 & 0.3590 & -0.23 & -0.07 \\
-0.3622 & 0.0233 & -0.11 & -0.18 & -0.3316 & 0.0705 & -0.06 & -0.29 & -0.2892 & 0.2426 & -0.15 & -0.18 \\
-0.4306 & 0.0000 & -0.21 & 0.00 & $-0.2743-0.1993$ & 0.17 & -0.24 & -0.3775 & 0.0000 & 0.00 & -0.24 \\
-0.3622 & -0.2328 & 0.11 & -0.18 & -0.1695 & -0.2936 & 0.26 & -0.15 & -0.3449 & -0.1535 & 0.10 & -0.22 \\
-0.1789 & -0.3917 & 0.19 & -0.09 & & & & & -0.1887 & -0.3269 & 0.21 & -0.12 \\
\hline
\end{tabular}

Table 11: Point loads applied in torsion for $M_{t}=1 \mathrm{nN} \cdot \mathrm{nm}$

the curvature of the nanotube.

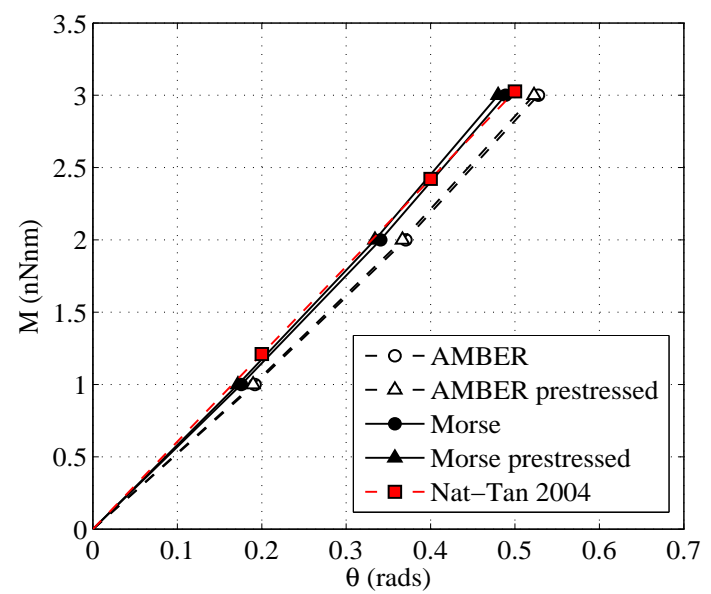

(a) $\mathrm{ZZ}(11,0)$ torsion

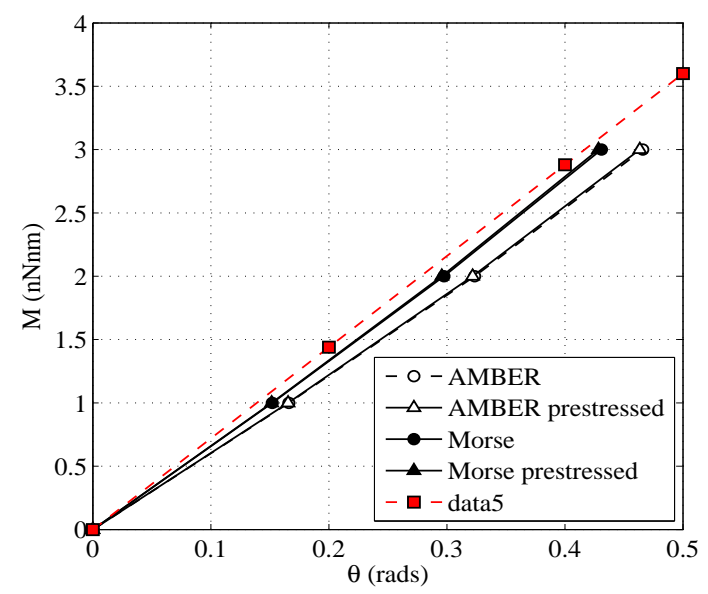

(b) $\mathrm{AC}(7,7)$ torsion

Figure 17: SWCNTs torsional behavior

The mechanical parameter chosen in this case for comparing values (with the same restraints aforementioned in bending) is the shear modulus $\mathrm{G}$, and it has been calculated as:

$$
G=\frac{M_{t} L}{J \varphi}
$$

where:

$M_{t}=$ torsional moment at the free end

$\varphi=$ rotation at the free end

$J=\quad$ polar inertia of the cross-section regarding the SWCNT as a hollow cylinder with $t=0.34 \mathrm{~nm}$

Averaging for each nanotube we can obtain the values of table 12

Shear modulus variation with respect to the nanotube diameter is depicted in figure 18 . Our results show same increasing trend reported by [21, fig 7] and [27, fig 10], but with smaller values (around 20\%) in the same way that Young modulus. Nevertheless, the magnitude order is reasonable and agrees well with these works. 


\begin{tabular}{lccccc}
\cline { 3 - 6 } & & \multicolumn{3}{c}{ AMBER AMBER MORSE MORSE } \\
\cline { 3 - 6 } & $\mathrm{d}(\mathrm{nm})$ & $\mathrm{G}(\mathrm{GPa})$ & $\mathrm{G}(\mathrm{GPa})$ & $\mathrm{G}(\mathrm{GPa})$ & $\mathrm{G}(\mathrm{GPa})$ \\
\hline $\mathrm{ZZ}(9,0)$ & 0.7046 & 261.74 & 266.22 & 284.62 & 293.15 \\
$\mathrm{ZZ}(11,0)$ & 0.8612 & 283.62 & 286.64 & 308.14 & 314.31 \\
$\mathrm{ZZ}(13,0)$ & 1.0178 & 292.91 & 295.15 & 318.70 & 323.42 \\
\hline $\mathrm{AC}(5,5)$ & 0.6780 & 235.67 & 240.04 & 255.05 & 260.29 \\
$\mathrm{AC}(7,7)$ & 0.9492 & 272.82 & 274.13 & 296.49 & 298.09 \\
$\mathrm{AC}(8,8)$ & 1.0848 & 282.88 & 283.91 & 307.83 & 310.15 \\
\hline $\mathrm{CH}(7,4)$ & 0.7541 & 413.04 & 224.00 & 185.06 & 304.67 \\
$\mathrm{CH}(8,5)$ & 0.8879 & 248.60 & 218.73 & 291.59 & 228.65 \\
$\mathrm{CH}(10,6)$ & 1.0960 & 269.80 & 260.86 & 306.30 & 251.50 \\
\hline
\end{tabular}

Table 12: Shear modulus SWCNTs in torsion

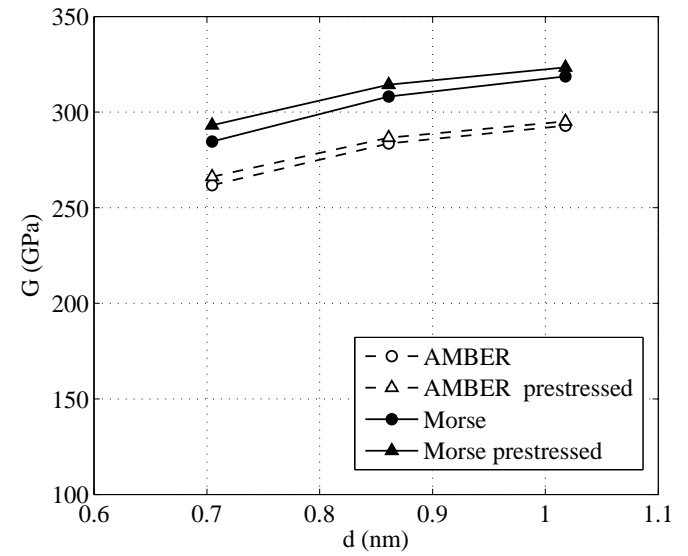

(a) ZigZag SWCNTs torsion

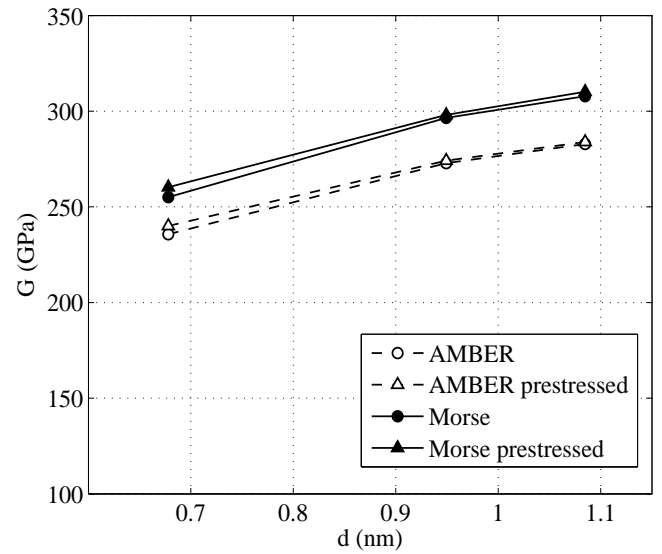

(b) Armchair SWCNTs torsion

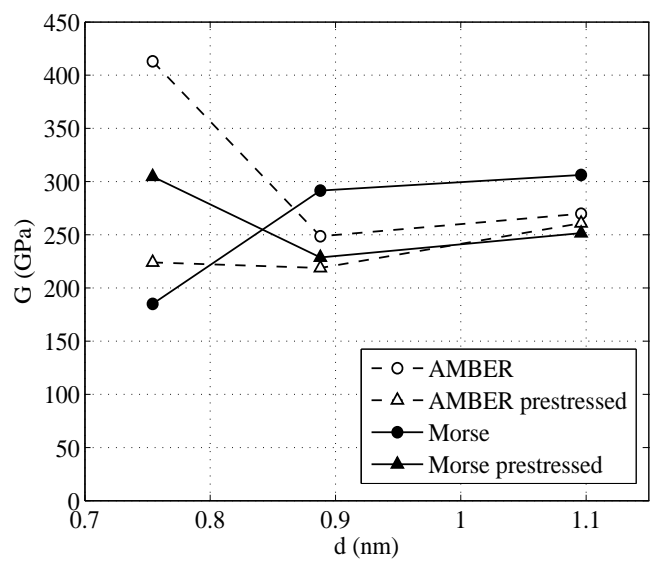

(c) Chiral SWCNTs torsion

Figure 18: Shear modulus SWCNTs in torsion 
Anyway, Morse potential seems to produce stiffer values of $G$ than AMBER potential, but it is not an important difference in our range of diameters. As can be seen in figure 18(c), ill-conditioning for Chiral nanotubes in torsion with Morse potential is also present. Likewise, the non-axisymmetry of Chiral nanotubes lead to eccentricities into the system of loads applied at the free end which cause bending; this bending-torsion coupling has a detrimental effect on final results (figure $19(\mathrm{c})$ ). As we mentioned before, this problem may be solved by means of imposed displacements instead of point loads at the free end.

Further understanding of the SWCNTs torsional behavior can be achieved from figure 19. Usual shortening can be seen in SWCNTs subjected to torsional loads if initial stresses are considered. Also, a radial deformation of the cross-section at the free end is observed with both potential functions as torsional moment increases (figure 19(b)). Since point loads are applied in tangential direction, they trend to move the atoms at the free end from their initial circular configuration. Therefore, those atoms pull out their neighbors and a conical deformation is rendered. This undesired deformation mode is the probable cause of the nonlinear response $M-\theta$ with linear potential (see figure 17).

Under torsional loads, we assumed independency of the chirality because of the known isotropy of the graphene layer into its own surface. Although our results seems to state that ZigZag nanotubes are stiffer subjected to torsion, the differences are not decisive.

\subsection{Derivation of Poisson's ratio}

As has been mentioned before, the continuum models are not applicable at the SWCNTs nano-scale size. In order to highlight this idea, values for the Poisson's ratio were obtained through the classical constitutive relationship:

$$
v=\frac{E}{2 G}-1
$$

involving the Young modulus obtained from the tensile simulations and the Shear modulus from the torsional ones. Output values are grouped in table 13 and their variation with respect to the nanotube diameter plotted in figure 20.

\begin{tabular}{lcccc}
\cline { 2 - 5 } & AMBER & $\begin{array}{c}\text { AMBER } \\
\text { prestress }\end{array}$ & MORSE & $\begin{array}{c}\text { MORSE } \\
\text { prestress }\end{array}$ \\
\cline { 2 - 5 } & $v$ & $v$ & $v$ & $v$ \\
\hline $\mathrm{ZZ}(9,0)$ & 0.48 & 0.82 & 0.58 & 1.02 \\
$\mathrm{ZZ}(11,0)$ & 0.38 & 0.57 & 0.48 & 0.71 \\
$\mathrm{ZZ}(13,0)$ & 0.35 & 0.47 & 0.44 & 0.59 \\
\hline $\mathrm{AC}(5,5)$ & 0.67 & 0.82 & 0.79 & 1.00 \\
$\mathrm{AC}(7,7)$ & 0.46 & 0.53 & 0.56 & 0.65 \\
$\mathrm{AC}(8,8)$ & 0.41 & 0.46 & 0.51 & 0.57 \\
\hline $\mathrm{CH}(7,4)$ & -0.06 & 0.89 & 1.45 & 0.71 \\
$\mathrm{CH}(8,5)$ & 0.58 & 0.90 & 0.54 & 1.23 \\
$\mathrm{CH}(10,6)$ & 0.45 & 0.56 & 0.48 & 0.95 \\
\hline
\end{tabular}

Table 13: Poisson's ration tension-torsion

Of course, the obtained results for Chiral nanotubes are not reliable because of the same numerical ill-conditioning problems found under torsion with Morse potential. Obviously, negative Poisson's ratio are senseless. On the other hand, from the ZigZag and Armchair results, we could state that Poisson's ratio decrease with increasing diameters up to an average value of about $0.25-0.3$. However, the remarkable scattering observed invalidates the last conclusion.

To sum up, equation (42) does not suitably render into the mechanical behavior of SWCNTs, underlining that continuum models are not capable of reproducing their discrete nature. Opposite to this idea Natsuki et. al [28] propose a value of $v=0.273$ and suggest the continuum body relationship (42) may be used for estimating the Poisson modulus of a planar graphene sheet. Nevertheless, their conclusion should not be extended to SWCNTs. 


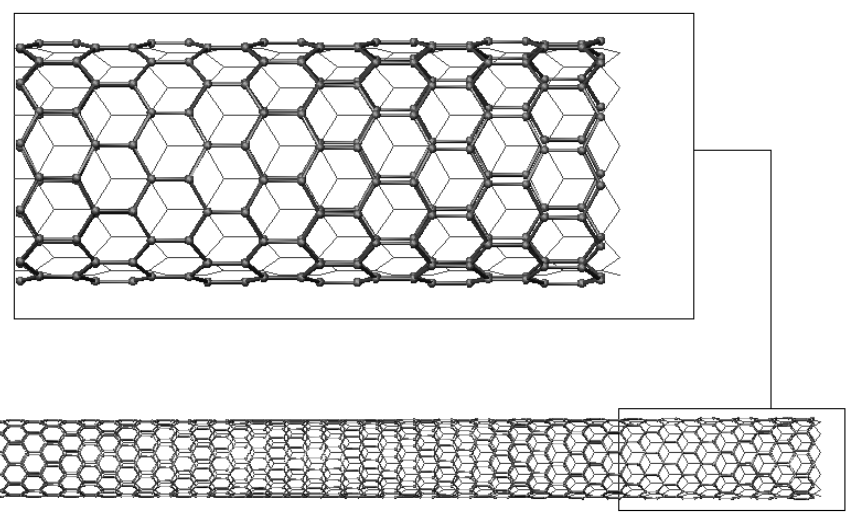

(a) ZigZag(11,0) $M=2 n N n m$, MORSE prestressed

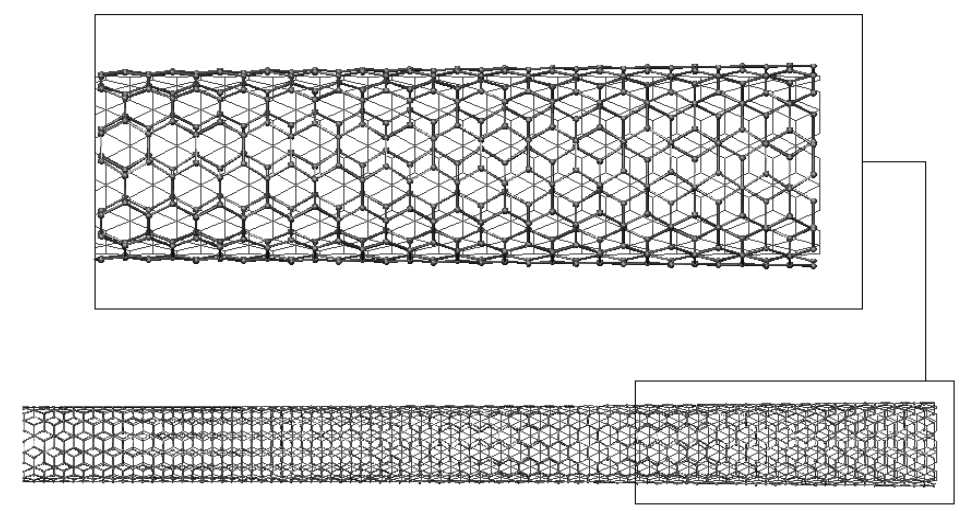

(b) Armchair(7,7) $M=3 n N n m$, AMBER prestressed

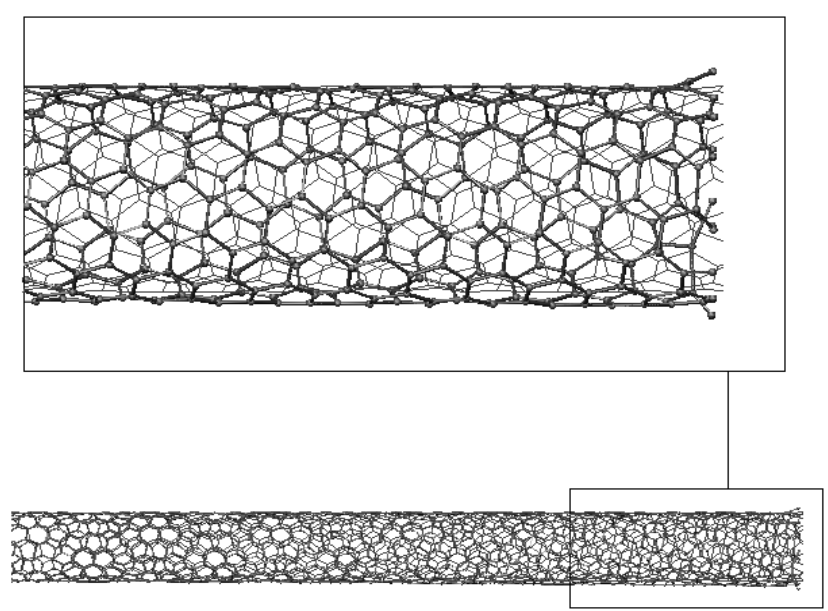

(c) $\operatorname{Chiral}(8,5) M=2 n N n m$, AMBER prestressed

Figure 19: Deformed shape SWCNTs torsion 


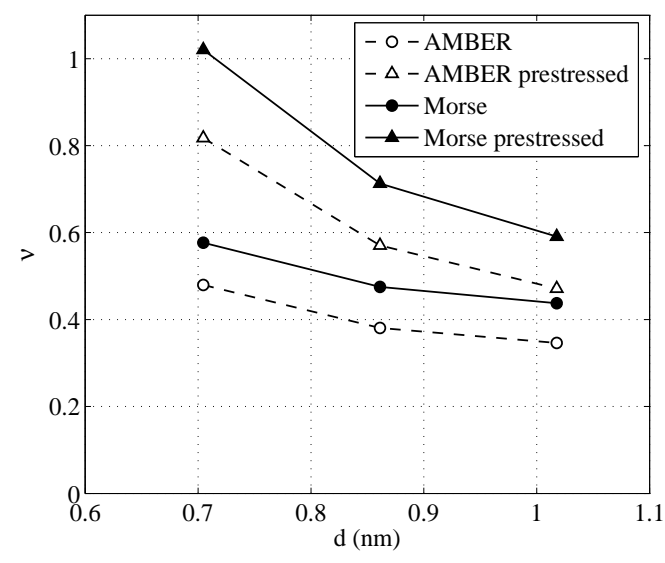

(a) ZigZag CNTs

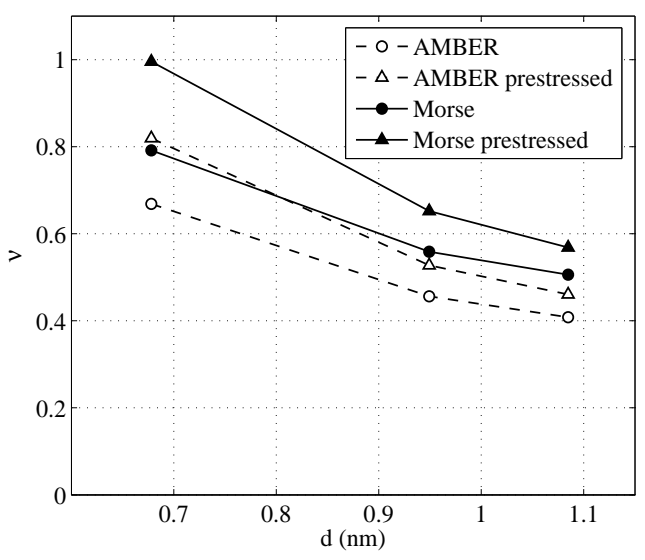

(b) Armchair CNTs

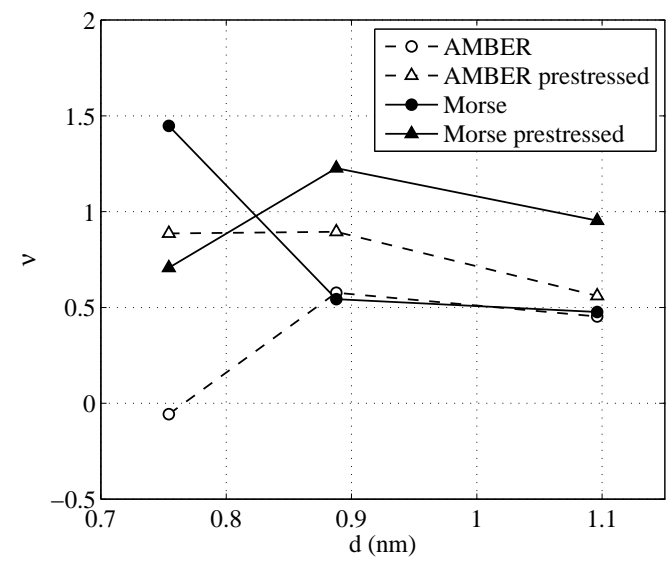

(c) Chiral CNTs

Figure 20: Variation of the Poisson's ratio with the diameter

\section{Concluding remarks}

In this paper, a new general formulation for the 'stick-spiral' model presented by Chang and Gao [26] has been presented. Calculations with both AMBER and Morse potential functions have been carried out, aimed to compare their final results. In addition, the effect of initial SWCNTs curvature has been introduced explicitly through a system of initial stresses (prestressed state) which contribute to maintain their circular cross-section.

Our formulation allows the model to be subjected to general load conditions with no need of additional equations, against other previous works [26], [27], [31] where a specific formulation is needed for each load case. As examples of this issue, four load configurations were adopted: tension, compression, bending and torsion of cantivelered SWCNTs. Numerical results of mechanical parameters and deformed shapes are presented and discussed.

Main conclusions obtained from our calculations are summarized as follows:

1. Morse potential provide stiffer results in tension than AMBER potential with differences over $15 \%$. The same stiffening effect produced by Morse function (15\% in compression, up to $13 \%$ in bending and around $8 \%$ in torsion) is present in all load cases. However, there are not important differences below strains about $6 \%$ in tension and below $4 \%$ in compression. 
2. Despite the nonlinear nature of the Morse potential, linear behavior under all load cases studied in this paper has been yielded. Of course, the forces updating through tangent stifnesses (fig 6 only may reproduce partially this nonlinearity, leading to an apparent contradiction. It should be noted that higher values of strains into each load case are not consistent with the model, which aims to reproduce the mechanical response of CNTs (geometrically linear) under small strains and displacements assumption.

3. Our new formulation is able to include initial stresses (preenergy) explicitly just by adding initial strains into the constitutive equation (19). This feature is a great novelty related to other works concerned with the 'stick-spiral' model [26], [27], [28], [30], [31]. The initial strains were adopted by keeping the carbon atoms into the cylinder surface of the nanotube at the undeformed shape, and regarding the initial location of the covalent bonds in a straight line linking two neighboring $\mathrm{C}$ atoms.

4. The prestressed state produces stiffening under tensile loads (about 5-18\%) and flexibilization under compressive loads (about $5-15 \%$ ). This phenomenon occurs because the prestressed state introduce radial resultant forces which tend to keep the circular cross-section of the nanotube. Thus, Poisson effect converts these radial forces into longitudinal shortening under any load case. Of course, the influence of the initial stresses into the axial behavior of SWCNTs should not be neglected.

On the other hand, the results are very similar in bending (differences in the range of 5\%) and torsion (differences around 3\%), and we can consider these load cases as independent of the prestressed state.

5. Despite the adoption of Young and shear moduli for the comparison of results, this choice may be controversial because of their high dependence on the wall thickness (which usually is taken as $0.34 \mathrm{~nm}$ ). Our only objective has been to validate our model with previous results, but not to establish quantitative mechanical parameters associated to a continuum.

6. In fact, is doubtful to deal with SWCNTs as a continuum body, as has been shown through the great scattering observed in the Poisson's ratio calculated by using eq. (42). Therefore, the classical constitutive relations do not suitably render a continuum equivalent medium for SWCNTs.

7. The application of standard homogenization theories [41], [42] could be very promising to treat the mechanical simulation of CNTs (although our paper is not directly related to these methods), given the specific characteristics of the problem (great number of unit cells which are repeated with geometric periodicity). Likewise, it would be taken tensile, compressive, flexural and torsional behavior as the preferred set of simple loading test cases for establishing the equivalent properties into the homogenization solution.

8. ZigZag SWCNTs are stiffer than Armchair ones under tensile loads (up to 8\%), but it is not clear the influence of the chirality on the final response under compressive, bending and torsional loads. It could be concluded the mechanical behavior of SWCNTs is almost independent of the chirality.

9. Young modulus $\mathrm{E}$ is decreasing with nanotube diameter in tension (only if prestressed state is included) and is increasing in compression. However, in compression, variation of Young modulus is much lower than in tension. Also, $\mathrm{E}$ is increasing under bending loads and shear modulus $\mathrm{G}$ is increasing under torsional loads with respect to the nanotube diameter. In both cases, Chiral results have not been taken into account due to the numerical ill-conditioning experimented for these results with Morse potential.

10. The non-axisymmetry of Chiral nanotubes lead to some bonds strongly tensioned and some other bonds strongly compressed under some of the load cases considered. Therefore, the very different stiffnesses from tension to compression with Morse longitudinal interaction causes ill-conditioning of the iterative procedure depicted in figure 6 and the results for Chiral CNTs are not completely reliable. Further improvements are currently in progress.

11. As output of our developed codes, deformed shapes, bond axial forces, angular moments and joint reactions are obtained. By interpreting the resulting deformed shapes, some undesired local deformations have been discovered at the cantilever free end, mainly with Chiral nanotubes. This effect is probably due to the local configuration of the bonds at the nanotube ends. Namely, some of the extreme bar elements in these zones are shorter than the real covalent $C-C$ bonds and the application of a set of point forces at the free end will trigger unexpected local deformations. However this problem may be removed by introducing imposed displacements at the free end by means of a set of moving pinned joints, which is currently being worked out. Anyway, the imposed displacements solutions may produce slightly different values of the mechanical parameters rendered (as shown by Ibrahimbegovic and Markovic [41] and against Markovic and Ibrahimvegovic [42]). In any case, local deformations near nanotube ends should be considered as local effects which could be readily neglected. 


\section{References}

[1] Iijima, S. Helical microtubules of graphitic carbon. Nature 1991; 354:56-58.

[2] Dresselhaus MS, Dresselhaus G, Avouris P. Carbon Nanotubes: Synthesis, Structure, Properties, and Applications. Berlin: Springer. 2001. Topics in Applied Physics, Vol 80.

[3] Rochefort A, Avouris P, Lesage F, Salahub DR. Electrical and mechanical properties of distorted carbon nanotubes. Phys. Rev. B 1999; 60(19):13824-830.

[4] Paulson S, Falvo MR, Snider N, Helser A, Hudson T, Seeger A et. al. In situ resistance measurements of strained carbon nanotubes. Applied Physics Letters 1999; 75(19): 2936-938.

[5] Hernández E, Goze C, Bernier P, Rubio A. Elastic properties of C and $B_{x} C_{y} N_{z}$ composite Nanotubes. Physical Review Letters 1998; $80: 4502-505$

[6] Krishnan A, Dujardin E, Ebbesen TW, Yianilos PN, Treacy MMJ. Young's modulus of single-walled nanotubes. Physical review B 1998; 58(20):14013-019.

[7] Popov VN, van Doren VE, Balkanski M. Elastic properties of single-walled carbon nanotubes. Physical Review B 2000; 61:3078-084.

[8] Lu JP. Elastic properties of carbon nanotubes and nanoropes. Phys. Rev. Lett. 1997; 79(7):1297-300.

[9] Belytschko T, Xiao SP, Schatz GC, Ruoff RS. Atomistic simulations of nanotube fracture. Phys. Rev. B 2002; 65(23):235430.

[10] Yu MF, Lourie O, Dyer MJ, Moloni K, Kelly TF, Ruoff RS. Strength and Breaking Mechanism of Multiwalled Carbon Nanotubes Under Tensile Load. Science 2000; 287(5453):637-40.

[11] Yu MF, Files BS, Arepalli S, Ruoff RS. Tensile loading of ropes of single wall carbon nanotubes and their mechanical properties. Phys. Rev. Lett. 2000; 84(24):5552-555.

[12] Wang MS, Kaplan-Ashiri I, Wei XL, Rosentsveig R, Wagner HD, Tenne R et. al. In situ tem measurements of the mechanical properties and behavior of $W S_{2}$ nanotubes. Nano Research 2008; 1(1):22-31.

[13] Chopra NG, Zettl A. Measurement of the elastic modulus of a multi-wall boron nitride nanotube. Solid State Communications 1998; 105(5):297-300.

[14] Wong EW, Sheehan PE, Lieber CM. Nanobeam mechanics: Elasticity, strength, and toughness of nanorods and nanotubes. Science 1997; 277(5334):1971-975.

[15] Falvo MR, Clary GJ, Taylor RM, Chi V, Brooks FP, Washburn S, et. al. Bending and buckling of carbon nanotubes under large strain. Nature 1997; 389(6651):582-84

[16] Salvetat JP, Briggs GAD, Bonard JM, Bacsa RR, Kulik AJ, Stöckli T, et. al. Elastic and shear moduli of single-walled carbon nanotube ropes. Phys. Rev. Lett. 1999; 82(5):944-47.

[17] Yakobson BI, Brabec CJ, Bernholc J. Nanomechanics of carbon tubes: Instabilities beyond linear response. Phys. Rev. Lett. 1996; 76(14):2511-514

[18] Li X, Yang W, Liu B. Bending Induced Rippling and Twisting of Multiwalled Carbon Nanotubes. Physical Review Letters 2007; 98(20):205502.

[19] Wang X, Wang X, Xiao J. A non-linear analysis of the bending modulus of carbon nanotubes with rippling deformations. Composite Structures 2005; 69(3):315-21.

[20] Meo M, Rossi M. Prediction of Young's modulus of single wall carbon nanotubes by molecular-mechanics based finite element modelling. Compos Sci Technol 2006; 66(11-12): 1597-605.

[21] Li C, Chou TW. A structural mechanics approach for the analysis of carbon nanotubes. International Journal of Solids and Structures 2003; 40(10):2487-499.

[22] Li C, Chou TW. Elastic moduli of multi-walled carbon nanotubes and the effect of van der waals forces. Composites Science and Technology 2003; 63(11):1517-524.

[23] Odegard GM, Gates TS, Nicholson LM, Wise KE. Equivalent-continuum modeling of nano-structured materials. Hampton, Virginia, USA, NASA/TM-2001-210863, 2001

[24] Zaeri MM, Ziaei-Rad S, Vahedi A, Karimzadeh F. Mechanical modelling of carbon nanomaterials from nanotubes to buckypaper. Carbon 2010; 48(13):3916-930

[25] Ibrahimbegovic A, Delaplace A. Microscale and mesoscale discrete models for dynamic fracture of structures built of brittle materials. Computers and Structures 2003; 81(12): 1255-1265.

[26] Chang T, Gao H. Size-dependent elastic properties of a single-walled carbon nanotube via a molecular mechanics model. Journal of the Mechanics and Physics of Solids 2003; 51(6):1059-074.

[27] Xiao J, Gama B, Gillespie Jr, J. An analytical molecular structural mechanics model for the mechanical properties of carbon nanotubes. International Journal of Solids and Structures 2005; 42(11-12):3075-092.

[28] Natsuki T, Tantrakarn K, Endo M. Prediction of elastic properties for singlewalled carbon nanotubes. Carbon 2004; 42(1):39-45.

[29] Wang Q. Effective in-plane stiffness and bending rigidity of armchair and zigzag carbon nanotubes. International Journal of Solids and Structures 2004; 41(20):5451-461.

[30] Natsuki T, Endo M. Stress simulation of carbon nanotubes in tension and compression. Carbon 2004; 42(11):2147-151.

[31] Chang T, Geng J, Guo X. Prediction of chirality- and size-dependent elastic properties of single-walled carbon nanotubes via a molecular mechanics model. Proc. of Royal Society A 2006; 462(2072):2523-40.

[32] Iijima S, Brabec C, Maiti A, Bernholc J. Structural flexibility of carbon nanotubes. Journal of Chemical Physics 1996; 104(5):2089-092.

[33] Jiang H, Zhang P, Liu B, Huang Y, Geubelle PH, Gao H, et. al. The effect of nanotube radius on the constitutive model for carbon nanotubes. Computational Materials Science 2003; 28(3-4):429-42.

[34] Pantano A, Parks DM, Boyce MC. Mechanics of deformation of single- and multi-wall carbon nanotubes. Journal of Mechanics Physics of Solids 2004; 52(4):789-821.

[35] Robertson DH, Brenner DW, Mintmire JW. Energetics of nanoscale graphitic tubules. Phys. Rev. B 1992; 45(21):12592-5. 
[36] Lau KT, Chipara M, Ling HY, Hui D. On the effective elastic moduli of carbon nanotubes for nanocomposite structures. Composites Part B: Engineering 2004; 35(2):95-101.

[37] Tersoff J. New empirical model for the structural properties of silicon. Phys. Rev. Lett. 1986; 56(6):632-5.

[38] Brenner DW. Empirical potential for hydrocarbons for use in simulating the chemical vapor deposition of diamond films. Phys. Rev. B 1990; 42(15):9458-71.

[39] WenXing B, ChangChun Z, WanZhao C. Simulation of young's modulus of singlewalled carbon nanotubes by molecular dynamics. Physica B: Condensed Matter 2004; 352(1-4):156-63.

[40] Arroyo M, Belytschko T. Nonlinear mechanical response and rippling of thick multiwalled carbon nanotubes. Phys. Rev. Lett. 2003; 91(21):215505-8

[41] Ibrahimvegovic A, Markovic D. Strong coupling methods in multi-phase and multi-scale modeling of inelastic behavior of heterogeneous structures. Computer Methods in Applied Mechanics and Engineering 2003; 192(28-30): 3089-3107.

[42] Markovic D, Ibrahimbegovic A. On micro-macro interface conditions for micro-scale based FEM for in elastic behavior of heterogeneous materials. Computer Methods in Applied Mechanics and Engineering 2004; 193(48-51): 5503-5523. 